\title{
DYNAMICAL LAWS OF THE COUPLED GROSS-PITAEVSKII EQUATIONS FOR SPIN-1 BOSE-EINSTEIN CONDENSATES*
}

\author{
WEIZHU $\mathrm{BAO}^{\dagger}$ AND YANZHI ZHANG ${ }^{\ddagger}$
}

\begin{abstract}
In this paper, we derive analytically the dynamical laws of the coupled GrossPitaevskii equations (CGPEs) without/with an angular momentum rotation term and an external magnetic field for modelling nonrotating/rotating spin-1 Bose-Eintein condensates. We prove the conservation of the angular momentum expectation when the external trapping potential is radially symmetric in two dimensions and cylindrically symmetric in three dimensions; obtain a system of first order ordinary differential equations (ODEs) governing the dynamics of the density of each component and solve the ODEs analytically in a few cases; derive a second order ODE for the dynamics of the condensate width and show that it is a periodic function without/with a perturbation; construct the analytical solution of the CGPEs when the initial data is chosen as a stationary state with its centerof-mass shifted away from the external trap center. Finally, these dynamical laws are confirmed by the direct numerical simulation results of the CGPEs.
\end{abstract}

Key words. Rotating spin-1 Bose-Einstein condensate, coupled Gross-Pitaevskii equations, angular momentum rotation, condensate width, angular momentum expectation.

AMS subject classifications. 35Q55, 65T99, 65Z05, 65N12, 65N35, 81-08

1. Introduction. The experimental realization of Bose-Einstein condensates (BECs) in magnetically trapped atomic gases at ultra-low temperatures $[1,12,16]$ has spurred great excitement in the atomic physics community and renewed the interest in studying the macroscopic quantum behavior of atoms. In earlier BEC experiments, the atoms were confined in a magnetic trap, in which the spin degree of freedom is frozen. The particles are described by a scalar model and the wave function of the particles is governed by the Gross-Pitaevskii equation (GPE) within the mean-field approximation [30, 18, 29]. One of the most important recent developments in BEC was the study of spin-1 and spin-2 condensates. In contrast to a single component BEC, a spin-F BEC is described by the coupled Gross-Pitaevskii equations (CGPEs) which consist of $2 F+1$ equations, each governing one of the $2 F+1$ hyperfine states $\left(m_{F}=-F,-F+1, \ldots, F-1, F\right)$ within the mean-field approximation [19, 28]. The spin-1 BEC was realized in experiments recently by using both ${ }^{23} \mathrm{Na}$ and ${ }^{87} \mathrm{Rb}$ $[23,31]$. In fact, the emergence of spin- $1 \mathrm{BEC}$ has created great opportunities for understanding degenerate gases with internal degrees of freedom $[19,5,6,13,15,20]$.

In this paper, we consider a rotating spin-1 BEC confined in an external trapping potential $V_{\text {ext }}(x, y, z)=\frac{m_{b}}{2}\left(\omega_{x}^{2} x^{2}+\omega_{y}^{2} y^{2}+\omega_{z}^{2} z^{2}\right)$ with $m_{b}$ the mass of BEC atoms, $\omega_{x}$, $\omega_{y}$ and $\omega_{z}$ the trapping frequencies in $x$-, $y$ - and $z$-direction, respectively. We assume that the interactions within the spin-1 BEC include the mean-field interaction with $c_{0}=4 \pi \hbar^{2}\left(a_{0}+2 a_{2}\right) / 3 m_{b}$ (positive for repulsive interaction and negative for attractive interaction) and the spin-exchange interaction with $c_{2}=4 \pi \hbar^{2}\left(a_{2}-a_{0}\right) / 3 m_{b}$ (positive for anti-ferromagnetic interaction and negative for ferromagnetic interaction) where $\hbar$ is the Planck constant, $a_{0}$ and $a_{2}$ are the $s$-wave scattering lengths for scattering channel of total hyperfine spin 0 (anti-parallel spin collision) and spin 2 (parallel spin collision), respectively. For temperatures below the critical temperature, the

\footnotetext{
*Received April 18, 2009; accepted for publication April 9, 2010.

${ }^{\dagger}$ Department of Mathematics and Center for Computational Science and Engineering, National University of Singapore, Singapore 117543 (bao@math.nus.edu.sg).

${ }^{\ddagger}$ Department of Scientific Computing, Florida State University, Tallahassee, FL 32306-4120, USA (yzhang5@fsu.edu).
} 
dynamics of the rotating spin-1 BEC is well described by the dimensionless GrossPitaevskii equation (GPE) with an angular momentum rotational term in the $d$ dimension $[19,6,5]$

$$
\begin{aligned}
i \partial_{t} \psi_{1}(\mathbf{x}, t)= & {\left[-\frac{1}{2} \nabla^{2}+V(\mathbf{x})+E_{1}-\Omega L_{z}+\beta_{n} \rho+\beta_{s}\left(\rho_{1}+\rho_{0}-\rho_{-1}\right)\right] \psi_{1} } \\
& +\beta_{s} \psi_{-1}^{*} \psi_{0}^{2}+B \psi_{0}, \\
i \partial_{t} \psi_{0}(\mathbf{x}, t)= & {\left[-\frac{1}{2} \nabla^{2}+V(\mathbf{x})+E_{0}-\Omega L_{z}+\beta_{n} \rho+\beta_{s}\left(\rho_{1}+\rho_{-1}\right)\right] \psi_{0} } \\
& +2 \beta_{s} \psi_{-1} \psi_{0}^{*} \psi_{1}+B\left(\psi_{1}+\psi_{-1}\right), \quad \mathbf{x} \in \mathbb{R}^{d}, \quad t>0 \\
i \partial_{t} \psi_{-1}(\mathbf{x}, t)= & {\left[-\frac{1}{2} \nabla^{2}+V(\mathbf{x})+E_{-1}-\Omega L_{z}+\beta_{n} \rho+\beta_{s}\left(\rho_{-1}+\rho_{0}-\rho_{1}\right)\right] \psi_{-1} } \\
& +\beta_{s} \psi_{1}^{*} \psi_{0}^{2}+B \psi_{0}, \quad \mathbf{x} \in \mathbb{R}^{d}, \quad j=-1,0,1
\end{aligned}
$$

Here, $\Psi=\Psi(\mathbf{x}, t):=\left(\psi_{1}(\mathbf{x}, t), \psi_{0}(\mathbf{x}, t), \psi_{-1}(\mathbf{x}, t)\right)^{T}$ is the dimensionless wave function of the rotating spin-1 BEC, $\rho_{j}=\rho_{j}(\mathbf{x}, t):=\left|\psi_{j}(\mathbf{x}, t)\right|^{2}$ is the density of the hyperfine spin component $m_{F}=j(j=-1,0,1)$ and $\rho=\rho_{1}+\rho_{0}+\rho_{-1}$ is the total density. $\Omega$ is the dimensionless angular momentum rotation speed, $E_{j} \in \mathbb{R}$ is the dimensionless Zeeman energy of spin component $m_{F}=j(j=-1,0,1)$ in the uniform external magnetic field, $B \in \mathbb{R}$ is the dimensionless external Ioffe-Pitchard magnetic field, and $\beta_{n}$ and $\beta_{s}$ are the dimensionless mean-field and spin-exchange interaction constants, respectively. $f^{*}$ denotes the conjugate of the function $f$ and $L_{z}$ is the $z$-component of the dimensionless angular momentum rotation defined as

$$
L_{z}=-i\left(x \partial_{y}-y \partial_{x}\right)=-i \partial_{\theta}:=-i \widehat{L}_{z} \quad \text { with } \quad \widehat{L}_{z}=\left(x \partial_{y}-y \partial_{x}\right)=\partial_{\theta},
$$

where $(r, \theta, z)$ is the cylindrical coordinates when $d=3$, and resp. $(r, \theta)$ is the polar coordinates when $d=2$. The above dimensionless quantities in three dimensions (3D) are obtained by scaling the length by the harmonic oscillator length $a_{s}=\sqrt{\hbar / m_{b} \omega}$, the time by $\omega^{-1}$ and the energy by $\hbar \omega$ with $\omega=\min \left\{\omega_{x}, \omega_{y}, \omega_{z}\right\}$. If $\Omega=0$, the CGPEs (1.1)-(1.3) describes a BEC in the nonrotating frame and $d$ can be chosen as $d=3,2$ or 1 ; on the contrary, if $\Omega \neq 0$, it is for a BEC in the rotating frame and $d$ can be chosen as $d=3$ or 2. In fact, the two-dimensional (2D) CGPEs can be viewed as a quasi-3D experimental setup with a strong confinement in the $z$-direction, i.e. $\omega_{y} \approx \omega_{x}$ and $\omega_{z} \gg \omega_{x}[9,6,8,7]$; and the one-dimensional (1D) CGPEs when $\Omega=0$ can be viewed as a quasi-3D experimental setup with strong confinement in both $y$ and $z$-directions, i.e. $\omega_{y} \gg \omega_{x}$ and $\omega_{z} \gg \omega_{x}[9,6,8,7]$. The dimensionless potential $V(\mathbf{x})$ takes the form

$$
V(\mathbf{x})=\frac{1}{2} \begin{cases}\gamma_{x}^{2} x^{2}, & d=1 \\ \gamma_{x}^{2} x^{2}+\gamma_{y}^{2} y^{2}, & d=2, \\ \gamma_{x}^{2} x^{2}+\gamma_{y}^{2} y^{2}+\gamma_{z}^{2} z^{2}, & d=3\end{cases}
$$

with $\gamma_{x}=\frac{\omega_{x}}{\omega}, \gamma_{y}=\frac{\omega_{y}}{\omega}, \gamma_{z}=\frac{\omega_{z}}{\omega}$. The parameters

$$
\beta_{n}=C_{d} \frac{4 \pi N\left(a_{0}+2 a_{2}\right)}{a_{s}}, \quad \beta_{s}=C_{d} \frac{4 \pi N\left(a_{2}-a_{0}\right)}{a_{s}},
$$


with $N$ the total number of particles in the spin-1 condensate and

$$
C_{d}= \begin{cases}\approx \sqrt{\gamma_{y} \gamma_{z}} / 2 \pi, & d=1 \\ \approx \sqrt{\gamma_{z} / 2 \pi}, & d=2 \\ 1, & d=3\end{cases}
$$

Two important invariants of the CGPEs (1.1)-(1.4) are the normalization of the wave function $[19,5]$

$$
\begin{aligned}
N_{\Psi}(t)=\|\Psi\|^{2} & :=\int_{\mathbb{R}^{d}}|\Psi(\mathbf{x}, t)|^{2} d \mathbf{x}=\int_{\mathbb{R}^{d}} \sum_{j=-1}^{1}\left|\psi_{j}(\mathbf{x}, t)\right|^{2} d \mathbf{x} \\
& \equiv \int_{\mathbb{R}^{d}} \sum_{j=-1}^{1}\left|\psi_{j}^{0}(\mathbf{x})\right|^{2} d \mathbf{x}=N_{\Psi}(0)=1, \quad t \geq 0
\end{aligned}
$$

and the energy per particle

$$
\begin{aligned}
E_{\Psi}(t)= & \int_{\mathbb{R}^{d}}\left[\sum_{j=-1}^{1}\left(\frac{1}{2}\left|\nabla \psi_{j}\right|^{2}+\left(V(\mathbf{x})+E_{j}\right)\left|\psi_{j}\right|^{2}-\Omega \operatorname{Re}\left(\psi_{j}^{*} L_{z} \psi_{j}\right)\right)\right. \\
& +\frac{\beta_{n}}{2} \rho_{0}^{2}+\frac{\beta_{n}+\beta_{s}}{2}\left(\rho_{1}^{2}+\rho_{-1}^{2}+2 \rho_{0}\left(\rho_{1}+\rho_{-1}\right)\right)+\left(\beta_{n}-\beta_{s}\right) \rho_{1} \rho_{-1} \\
& \left.+2 \beta_{s} \operatorname{Re}\left(\psi_{-1}^{*} \psi_{0}^{2} \psi_{1}^{*}\right)+2 B \operatorname{Re}\left(\psi_{0}^{*}\left(\psi_{1}+\psi_{-1}\right)\right)\right] d \mathbf{x} \equiv E_{\Psi}(0), \quad t \geq 0
\end{aligned}
$$

where $\operatorname{Re}(f)$ denotes the real part of the function $f$. In addition, when $B=0$, another important invariant is the total magnetization $[19,5,6]$

$$
\begin{aligned}
M_{\Psi}(t) & =\int_{\mathbb{R}^{d}} \sum_{j=-1}^{1} j\left|\psi_{j}(\mathbf{x}, t)\right|^{2} d \mathbf{x}=\int_{\mathbb{R}^{d}}\left[\left|\psi_{1}(\mathbf{x}, t)\right|^{2}-\left|\psi_{-1}(\mathbf{x}, t)\right|^{2}\right] d \mathbf{x} \\
& \equiv \int_{\mathbb{R}^{d}}\left[\left|\psi_{1}^{0}(\mathbf{x})\right|^{2}-\left|\psi_{-1}^{0}(\mathbf{x})\right|^{2}\right] d \mathbf{x}=M_{\Psi}(0), \quad t \geq 0
\end{aligned}
$$

with $-1 \leq M_{\Psi}(0) \leq 1$

In the effort of exploring the rich properties of spinor dynamics, various numerical and theoretical studies have been carried out in the last few years. From a numerical point of view, different efficient and accurate numerical methods have been proposed for computing the ground state and dynamics of spin-1 BEC (cf. [38, 39, 9, 6, 5, 34] and references therein). From a theoretical point of view, different properties of spin-1 BEC have been investigated, e.g. the coreless vortices [24, 13], the quantum tunneling phenomena in double well potential [27], the interaction of soliton solutions [33, 14], single mode approximation (SMA) and coherent dynamics [22, 25], the effect of finite temperature in the context of Bogoliubov-de-Gennes framework [26] and so on. To our knowledge, there are very few rigorous mathematical results for the CGPEs (1.1)-(1.3) of the dynamics of spin-1 BEC. The aim of this paper is to study mathematically the dynamical properties of spin-1 BEC based on the CGPEs (1.1)-(1.3) including time evolution of the density of each component, expected value of angular momentum (or angular momentum expectation) and condensate width as well as the construction of an analytical solution when the initial data is chosen as the stationary state with its 
center-of-mass shifted from the trap center. We remark that similar mathematical results have been carried out recently for GPE of single-component BEC [10,3] and CGPEs of two-component BEC [37, 2].

The paper is organized as follows. In Section 2, we derive a system of first-order ordinary differential equations (ODEs) for the dynamics of the density of each component and other related quantities. The ODE system is analytically solved for a few cases. In Section 3, we prove the conservation of the angular momentum expectation when the external trap is radially symmetric in $2 \mathrm{D}$ and cylindrically symmetric in 3D. In Section 4, we obtain a second-order ODE for the dynamics of the condensate width and show that the condensate width is a periodic function in 1D without nonlinearity and in $2 \mathrm{D}$ when the trap is radially symmetric. An analytical solution is constructed for the CGPEs when the initial data is chosen as a stationary state with its center-ofmass shifted from the trap center in Section 5. In Section 6, some numerical results on the dynamics of spin-1 BEC are reported to confirm the analytical results. Some concluding remarks are drawn in Section 7.

2. Dynamics of the mass of each component. Define the mass (or density) of the spin component $m_{F}=j$ as

$$
N_{j}(t):=\int_{\mathbb{R}^{d}}\left|\psi_{j}(\mathbf{x}, t)\right|^{2} d \mathbf{x}, \quad t \geq 0, \quad j=-1,0,1
$$

It is easy to see that, when $\beta_{s}=0$ and $B=0$ in the CGPEs (1.1)-(1.3), the mass of each spin component is conserved, i.e.

$$
N_{j}(t)=\int_{\mathbb{R}^{d}}\left|\psi_{j}(\mathbf{x}, t)\right|^{2} d \mathbf{x} \equiv \int_{\mathbb{R}^{d}}\left|\psi_{j}^{0}(\mathbf{x})\right|^{2} d \mathbf{x}:=N_{j}^{(0)}, \quad t \geq 0, \quad j=-1,0,1 .
$$

In order to get a closed system for the above quantities, we also define the following quantities which may have physical meaning but we will not discuss it here for brevity:

$$
\begin{aligned}
& P_{l, k}(t)=i \int_{\mathbb{R}^{d}}\left[\psi_{l}^{*}(\mathbf{x}, t) \psi_{k}(\mathbf{x}, t)-\psi_{l}(\mathbf{x}, t) \psi_{k}^{*}(\mathbf{x}, t)\right] d \mathbf{x}, \\
& Q_{l, k}(t)=\int_{\mathbb{R}^{d}}\left[\psi_{l}^{*}(\mathbf{x}, t) \psi_{k}(\mathbf{x}, t)+\psi_{l}(\mathbf{x}, t) \psi_{k}^{*}(\mathbf{x}, t)\right] d \mathbf{x}, \quad-1 \leq l<k \leq 1, \\
& P(t)=P_{-1,0}(t)+P_{0,1}(t), \quad Q(t)=Q_{-1,0}(t)+Q_{0,1}(t), \quad t \geq 0,
\end{aligned}
$$

and denote

$$
p_{0}=\frac{E_{1}-E_{-1}}{2}, \quad q_{0}=\frac{E_{1}+E_{-1}-2 E_{0}}{2} .
$$

For the dynamics of the mass (or density) of each component, we have the following lemmas:

Lemma 2.1. Suppose $\Psi(\mathbf{x}, t)$ is the solution of the CGPEs (1.1)-(1.4), then we 
have

$N_{1}^{\prime}(t)-B P_{0,1}(t)=F_{1}(t)$

$N_{0}^{\prime}(t)+B\left[P_{0,1}(t)-P_{-1,0}(t)\right]=-2 F_{1}(t)$,

$N_{-1}^{\prime}(t)+B P_{-1,0}(t)=F_{1}(t)$

$P_{0,1}^{\prime}(t)-2 B\left[N_{0}(t)-N_{1}(t)\right]+B Q_{-1,1}(t)-\left(E_{1}-E_{0}\right) Q_{0,1}(t)=F_{2}(t)$,

$P_{-1,0}^{\prime}(t)+2 B\left[N_{0}(t)-N_{-1}(t)\right]-B Q_{-1,1}(t)+\left(E_{-1}-E_{0}\right) Q_{-1,0}(t)=-F_{2}(t)$,

$P_{-1,1}^{\prime}(t)-B\left[Q_{-1,0}(t)-Q_{0,1}(t)\right]-\left(E_{1}-E_{-1}\right) Q_{-1,1}(t)=F_{3}(t)$,

$Q_{0,1}^{\prime}(t)-B P_{-1,1}(t)+\left(E_{1}-E_{0}\right) P_{0,1}(t)=F_{4}(t)$,

$Q_{-1,0}^{\prime}(t)+B P_{-1,1}(t)-\left(E_{-1}-E_{0}\right) P_{-1,0}(t)=-F_{4}(t)$,

$Q_{-1,1}^{\prime}(t)-B\left[P_{0,1}(t)-P_{-1,0}(t)\right]+\left(E_{1}-E_{-1}\right) P_{-1,1}(t)=F_{5}(t), \quad t \geq 0$,

with initial conditions

$N_{j}(0)=\int_{\mathbb{R}^{d}}\left|\psi_{j}^{0}(\mathbf{x})\right|^{2} d \mathbf{x}:=N_{j}^{(0)}, \quad j=-1,0,1$,

$P_{l, k}(0)=i \int_{\mathbb{R}^{d}}\left[\left(\psi_{l}^{0}(\mathbf{x})\right)^{*} \psi_{k}^{0}(\mathbf{x})-\psi_{l}^{0}(\mathbf{x})\left(\psi_{k}^{0}(\mathbf{x})\right)^{*}\right] d \mathbf{x}:=P_{l, k}^{(0)}$,

$Q_{l, k}(0)=\int_{\mathbb{R}^{d}}\left[\left(\psi_{l}^{0}(\mathbf{x})\right)^{*} \psi_{k}^{0}(\mathbf{x})+\psi_{l}^{0}(\mathbf{x})\left(\psi_{k}^{0}(\mathbf{x})\right)^{*}\right] d \mathbf{x}:=Q_{l, k}^{(0)}, \quad-1 \leq l<k \leq 1$,

and

$$
\begin{aligned}
& F_{1}(t)=2 \beta_{s} \int_{\mathbb{R}^{d}} \operatorname{Im}\left(\psi_{-1}^{*} \psi_{0}^{2} \psi_{1}^{*}\right) d \mathbf{x}, \quad t \geq 0, \\
& F_{2}(t)=2 \beta_{s} \int_{\mathbb{R}^{d}} \operatorname{Re}\left[\rho_{0} \psi_{0}\left(\psi_{1}^{*}+\psi_{-1}^{*}\right)-2\left(\rho_{-1} \psi_{0} \psi_{1}^{*}+\rho_{1} \psi_{0} \psi_{-1}^{*}\right)\right] d \mathbf{x}, \\
& F_{3}(t)=2 \beta_{s} \int_{\mathbb{R}^{d}} \operatorname{Re}\left[2\left(\rho_{1}-\rho_{-1}\right) \psi_{1} \psi_{-1}^{*}+\left(\psi_{0}^{*}\right)^{2}\left(\psi_{-1}^{2}-\psi_{1}^{2}\right)\right] d \mathbf{x}, \\
& F_{4}(t)=2 \beta_{s} \int_{\mathbb{R}^{d}} \operatorname{Im}\left[\rho_{0} \psi_{0}^{*}\left(\psi_{1}-\psi_{-1}\right)-2\left(\rho_{-1} \psi_{0}^{*} \psi_{1}-\rho_{1} \psi_{0}^{*} \psi_{-1}\right)\right] d \mathbf{x}, \\
& F_{5}(t)=2 \beta_{s} \int_{\mathbb{R}^{d}} \operatorname{Im}\left[2\left(\rho_{1}-\rho_{-1}\right) \psi_{-1}^{*} \psi_{1}-\left(\psi_{0}^{*}\right)^{2}\left(\psi_{1}^{2}+\psi_{-1}^{2}\right)\right] d \mathbf{x},
\end{aligned}
$$

where $\operatorname{Im}(f)$ denotes the imaginary part of the function $f$.

Proof. Differentiating (2.1) with respect to $t$ for $j=1$, integrating by parts and 
noticing (1.1)-(1.3), we obtain

$$
\begin{aligned}
N_{1}^{\prime}(t)= & \int_{\mathbb{R}^{d}}\left(\psi_{1} \partial_{t} \psi_{1}^{*}+\psi_{1}^{*} \partial_{t} \psi_{1}\right) d \mathbf{x}=i \int_{\mathbb{R}^{d}}\left[\left(-i \partial_{t} \psi_{1}^{*}\right) \psi_{1}-\psi_{1}^{*}\left(i \partial_{t} \psi_{1}\right)\right] d \mathbf{x} \\
= & i \int_{\mathbb{R}^{d}}\left[\left(\frac{1}{2}\left|\nabla \psi_{1}\right|^{2}+\left(V(\mathbf{x})+E_{1}\right)\left|\psi_{1}\right|^{2}-i \Omega \psi_{1} \widehat{L}_{z} \psi_{1}^{*}+\beta_{n} \rho\left|\psi_{1}\right|^{2}\right.\right. \\
& \left.+\beta_{s}\left(\rho_{1}+\rho_{0}-\rho_{-1}\right)\left|\psi_{1}\right|^{2}+\beta_{s} \psi_{1}\left(\psi_{0}^{*}\right)^{2} \psi_{-1}+B \psi_{1} \psi_{0}^{*}\right) \\
& -\left(\frac{1}{2}\left|\nabla \psi_{1}\right|^{2}+\left(V(\mathbf{x})+E_{1}\right)\left|\psi_{1}\right|^{2}+i \Omega \psi_{1}^{*} \widehat{L}_{z} \psi_{1}+\beta_{n} \rho\left|\psi_{1}\right|^{2}\right. \\
& \left.\left.+\beta_{s}\left(\rho_{1}+\rho_{0}-\rho_{-1}\right)\left|\psi_{1}\right|^{2}+\beta_{s} \psi_{1}^{*} \psi_{0}^{2} \psi_{-1}^{*}+B \psi_{1}^{*} \psi_{0}\right)\right] d \mathbf{x} \\
= & i B \int_{\mathbb{R}^{d}}\left(\psi_{0}^{*} \psi_{1}-\psi_{1}^{*} \psi_{0}\right) d \mathbf{x}+i \beta_{s} \int_{\mathbb{R}^{d}}\left(\psi_{1}\left(\psi_{0}^{*}\right)^{2} \psi_{-1}-\psi_{1}^{*} \psi_{0}^{2} \psi_{-1}^{*}\right) d \mathbf{x} .
\end{aligned}
$$

Thus (2.7) is a combination of (2.24), (2.3) and (2.19); we can get (2.8) and (2.9) in a similar manner. Differentiating (2.3) with respect to $t$ for $l=0$ and $k=1$, integrating by parts and noticing (1.1)-(1.3), we have

$$
\begin{aligned}
& P_{0,1}^{\prime}(t) \\
= & i \int_{\mathbb{R}^{d}}\left(\psi_{0}^{*} \partial_{t} \psi_{1}+\psi_{1} \partial_{t} \psi_{0}^{*}-\psi_{0} \partial_{t} \psi_{1}^{*}-\psi_{1}^{*} \partial_{t} \psi_{0}\right) d \mathbf{x} \\
= & \int_{\mathbb{R}^{d}}\left[\psi_{0}^{*}\left(-\frac{1}{2} \nabla^{2}+V(\mathbf{x})+E_{1}-\Omega L_{z}+\beta_{n} \rho+\beta_{s}\left(\rho_{1}+\rho_{0}-\rho_{-1}\right)\right) \psi_{1}\right. \\
& -\psi_{1}\left(-\frac{1}{2} \nabla^{2}+V(\mathbf{x})+E_{0}-\Omega L_{z}^{*}+\beta_{n} \rho+\beta_{s}\left(\rho_{1}+\rho_{-1}\right)\right) \psi_{0}^{*} \\
& +\psi_{0}\left(-\frac{1}{2} \nabla^{2}+V(\mathbf{x})+E_{1}-\Omega L_{z}^{*}+\beta_{n} \rho+\beta_{s}\left(\rho_{1}+\rho_{0}-\rho_{-1}\right)\right) \psi_{1}^{*} \\
& -\psi_{1}^{*}\left(-\frac{1}{2} \nabla^{2}+V(\mathbf{x})+E_{0}-\Omega L_{z}+\beta_{n} \rho+\beta_{s}\left(\rho_{1}+\rho_{-1}\right)\right) \psi_{0} \\
& +\beta_{s}\left|\psi_{0}\right|^{2} \psi_{-1}^{*} \psi_{0}+B\left|\psi_{0}\right|^{2}-2 \beta_{s}\left|\psi_{1}\right|^{2} \psi_{-1}^{*} \psi_{0}-B \psi_{1}\left(\psi_{1}^{*}+\psi_{-1}^{*}\right) \\
& \left.+\beta_{s}\left|\psi_{0}\right|^{2} \psi_{-1} \psi_{0}^{*}+B\left|\psi_{0}\right|^{2}-2 \beta_{s}\left|\psi_{1}\right|^{2} \psi_{-1} \psi_{0}^{*}-B \psi_{1}^{*}\left(\psi_{1}+\psi_{-1}\right)\right] d \mathbf{x} \\
= & \int_{\mathbb{R}^{d}}\left[2 B\left(\left|\psi_{0}\right|^{2}-\left|\psi_{1}\right|^{2}\right)+\left(E_{1}-E_{0}\right)\left(\psi_{0}^{*} \psi_{1}+\psi_{0} \psi_{1}^{*}\right)-B\left(\psi_{-1}^{*} \psi_{1}+\psi_{-1} \psi_{1}^{*}\right)\right. \\
& \left.+\beta_{s}\left(\rho_{0}-2 \rho_{-1}\right)\left(\psi_{0} \psi_{1}^{*}+\psi_{0}^{*} \psi_{1}\right)+\beta_{s}\left(\rho_{0}-2 \rho_{1}\right)\left(\psi_{-1} \psi_{0}^{*}+\psi_{-1}^{*} \psi_{0}\right)\right] d \mathbf{x} . \quad(2.25)
\end{aligned}
$$

Thus (2.10) is a combination of (2.25), (2.3), (2.4) and (2.20); we can get (2.11) and (2.12) in a similar manner. Differentiating (2.4) with respect to $t$ for $l=0$ and $k=1$, 
integrating by parts and noticing (1.1)-(1.3), we have

$$
\begin{aligned}
Q_{0,1}^{\prime}(t)= & \int_{\mathbb{R}^{d}}\left(\psi_{0}^{*} \partial_{t} \psi_{1}+\psi_{1} \partial_{t} \psi_{0}^{*}+\psi_{0} \partial_{t} \psi_{1}^{*}+\psi_{1}^{*} \partial_{t} \psi_{0}\right) d \mathbf{x} \\
= & i \int_{\mathbb{R}^{d}}\left[-\psi_{0}^{*}\left(-\frac{1}{2} \nabla^{2}+V(\mathbf{x})+E_{1}-\Omega L_{z}+\beta_{n} \rho+\beta_{s}\left(\rho_{1}+\rho_{0}-\rho_{-1}\right)\right) \psi_{1}\right. \\
& +\psi_{1}\left(-\frac{1}{2} \nabla^{2}+V(\mathbf{x})+E_{0}-\Omega L_{z}^{*}+\beta_{n} \rho+\beta_{s}\left(\rho_{1}+\rho_{-1}\right)\right) \psi_{0}^{*} \\
& +\psi_{0}\left(-\frac{1}{2} \nabla^{2}+V(\mathbf{x})+E_{1}-\Omega L_{z}^{*}+\beta_{n} \rho+\beta_{s}\left(\rho_{1}+\rho_{0}-\rho_{-1}\right)\right) \psi_{1}^{*} \\
& -\psi_{1}^{*}\left(-\frac{1}{2} \nabla^{2}+V(\mathbf{x})+E_{0}-\Omega L_{z}+\beta_{n} \rho+\beta_{s}\left(\rho_{1}+\rho_{-1}\right)\right) \psi_{0} \\
& -\beta_{s}\left|\psi_{0}\right|^{2} \psi_{-1}^{*} \psi_{0}-B\left|\psi_{0}\right|^{2}+2 \beta_{s}\left|\psi_{1}\right|^{2} \psi_{-1}^{*} \psi_{0}+B \psi_{1}\left(\psi_{1}^{*}+\psi_{-1}^{*}\right) \\
& \left.+\beta_{s}\left|\psi_{0}\right|^{2} \psi_{-1} \psi_{0}^{*}+B\left|\psi_{0}\right|^{2}-2 \beta_{s}\left|\psi_{1}\right|^{2} \psi_{-1} \psi_{0}^{*}-B \psi_{1}^{*}\left(\psi_{1}+\psi_{-1}\right)\right] d \mathbf{x} \\
= & i \int_{\mathbb{R}^{d}}\left[\left(E_{1}-E_{0}\right)\left(\psi_{0} \psi_{1}^{*}-\psi_{0}^{*} \psi_{1}\right)+B\left(\psi_{-1}^{*} \psi_{1}-\psi_{-1} \psi_{1}^{*}\right)\right. \\
& +2 \beta_{s}\left|\psi_{1}\right|^{2}\left(\psi_{-1}^{*} \psi_{0}-\psi_{-1} \psi_{0}^{*}\right)+2 \beta_{s} \rho_{-1}\left(\psi_{0}^{*} \psi_{1}-\psi_{0} \psi_{1}^{*}\right) \\
& \left.-\beta_{s} \rho_{0}\left(\psi_{0}^{*} \psi_{1}-\psi_{0} \psi_{1}^{*}-\psi_{0}^{*} \psi_{-1}+\psi_{0} \psi_{-1}^{*}\right)\right] d \mathbf{x} .
\end{aligned}
$$

Thus (2.13) is a combination of (2.26), (2.3), (2.4) and (2.22), and we can get (2.14) and (2.15) in a similar manner.

Lemma 2.2. Suppose that $\Psi(\mathbf{x}, t)$ is the solution of the CGPEs (1.1)-(1.4) and $q_{0}=\left(E_{1}+E_{-1}-2 E_{0}\right) / 2=0$; then we have

$$
\begin{aligned}
& M_{\Psi}(t)=M_{\Psi}^{(0)}+\frac{B}{\mu_{1}}\left[P^{(0)} \sin \left(\mu_{1} t\right)+C_{1}\left(1-\cos \left(\mu_{1} t\right)\right)\right], \\
& P(t)=P^{(0)} \cos \left(\mu_{1} t\right)+C_{1} \sin \left(\mu_{1} t\right), \quad t \geq 0, \\
& Q(t)=Q^{(0)}-\frac{p_{0}}{\mu_{1}}\left[P^{(0)} \sin \left(\mu_{1} t\right)+C_{1}\left(1-\cos \left(\mu_{1} t\right)\right)\right],
\end{aligned}
$$

where

$$
\mu_{1}=\sqrt{2 B^{2}+p_{0}^{2}}, \quad C_{1}=\frac{p_{0} Q^{(0)}-2 B M_{\Psi}^{(0)}}{\mu_{1}} .
$$

The above analytical solution immediately implies that: (i) the total magnetization $M_{\Psi}(t)$ is conserved if $B=0$; otherwise, it is a periodic function with period $T=$ $\frac{2 \pi}{\sqrt{2 B^{2}+p_{0}^{2}}}=\frac{4 \pi}{\sqrt{8 B^{2}+\left(E_{1}-E_{-1}\right)^{2}}}$ if $B \neq 0$; (ii) $P(t)$ and $Q(t)$ are conserved if $B=0$ and $p_{0}=\left(E_{1}-E_{-1}\right) / 2=0$; otherwise, they are periodic functions with the period $T$ if either $B \neq 0$ or $p_{0}=\left(E_{1}-E_{-1}\right) / 2 \neq 0$.

Proof. Subtracting (2.9) from (2.7) and noticing (1.11) and (2.5), we obtain

$$
M_{\Psi}^{\prime}(t)-B P(t)=0, \quad t \geq 0 .
$$

Summing (2.10) and (2.11), noticing (1.11), (2.6) and (2.5), we have

$$
P^{\prime}(t)+2 B M_{\Psi}(t)-p_{0} Q(t)=0, \quad t \geq 0 .
$$


Similarly, summing (2.13) and (2.14), noticing (2.5) and (2.6), we get

$$
Q^{\prime}(t)+p_{0} P(t)=0, \quad t \geq 0 .
$$

Solving the above ODEs (2.30), (2.31) and (2.32) with the initial conditions (2.16), (2.17) and (2.18) immediately gives the analytical solution (2.27)-(2.29).

Lemma 2.3. Suppose that $\Psi(\mathbf{x}, t)$ is the solution of the CGPEs (1.1)-(1.4) and $\beta_{s}=0$, then there are

(i) If $B=0$, we have

$$
\begin{aligned}
& N_{1}(t) \equiv N_{1}^{(0)}, \quad N_{0}(t) \equiv N_{0}^{(0)}, \quad N_{-1}(t) \equiv N_{-1}^{(0)}, \\
& P_{0,1}(t)=P_{0,1}^{(0)} \cos \left(\left(E_{1}-E_{0}\right) t\right)+Q_{0,1}^{(0)} \sin \left(\left(E_{1}-E_{0}\right) t\right), \\
& Q_{0,1}(t)=Q_{0,1}^{(0)} \cos \left(\left(E_{1}-E_{0}\right) t\right)-P_{0,1}^{(0)} \sin \left(\left(E_{1}-E_{0}\right) t\right), \\
& P_{-1,0}(t)=P_{-1,0}^{(0)} \cos \left(\left(E_{-1}-E_{0}\right) t\right)-Q_{-1,0}^{(0)} \sin \left(\left(E_{-1}-E_{0}\right) t\right), \\
& Q_{-1,0}(t)=Q_{-1,0}^{(0)} \cos \left(\left(E_{-1}-E_{0}\right) t\right)+P_{-1,0}^{(0)} \sin \left(\left(E_{-1}-E_{0}\right) t\right), \\
& P_{-1,1}(t)=P_{-1,1}^{(0)} \cos \left(2 p_{0} t\right)+Q_{-1,1}^{(0)} \sin \left(2 p_{0} t\right), \\
& Q_{-1,1}(t)=Q_{-1,1}^{(0)} \cos \left(2 p_{0} t\right)-P_{-1,1}^{(0)} \sin \left(2 p_{0} t\right), \quad t \geq 0 .
\end{aligned}
$$

The analytical solutions suggest that: (a) the mass (or density) of each component $N_{j}(t)(j=-1,0,1)$ is conserved; (b) for $-1 \leq l<k \leq 1$, the quantities $P_{l, k}(t)$ and $Q_{l, k}(t)$ are conserved if $E_{l}=E_{k}$; otherwise, they are periodic functions with period $T=2 \pi /\left|E_{l}-E_{k}\right|$ if $E_{l} \neq E_{k}$.

(ii) If $E_{1}=E_{0}=E_{-1}$ (i.e., $p_{0}=q_{0}=0$ ), we have

$$
\begin{aligned}
& N_{1}(t)= C_{1}+C_{2} \cos \left(\lambda_{1} t\right)+\frac{P^{(0)}}{2 \sqrt{2}} \sin \left(\lambda_{1} t\right) \\
&+\frac{C_{3}}{4 \sqrt{2}} \sin \left(\lambda_{2} t\right)+\frac{C_{5}}{4 \sqrt{2}}\left(1-\cos \left(\lambda_{2} t\right)\right), \\
& N_{0}(t)=1-2 C_{1}-\frac{C_{3}}{2 \sqrt{2}} \sin \left(\lambda_{2} t\right)-\frac{C_{5}}{2 \sqrt{2}}\left(1-\cos \left(\lambda_{2} t\right)\right), \\
& N_{-1}(t)=C_{1}-C_{2} \cos \left(\lambda_{1} t\right)-\frac{P^{(0)}}{2 \sqrt{2}} \sin \left(\lambda_{1} t\right) \\
&+\frac{C_{3}}{4 \sqrt{2}} \sin \left(\lambda_{2} t\right)+\frac{C_{5}}{4 \sqrt{2}}\left(1-\cos \left(\lambda_{2} t\right)\right), \\
& P_{0,1}(t)=\frac{C_{3}}{2} \cos \left(\lambda_{2} t\right)+\frac{C_{5}}{2} \cos \left(\lambda_{2} t\right)-\sqrt{2} C_{2} \sin \left(\lambda_{1} t\right)+\frac{P^{(0)}}{2} \cos \left(\lambda_{1} t\right), \\
& P_{-1,0}(t)=-\frac{C_{3}}{2} \cos \left(\lambda_{2} t\right)-\frac{C_{5}}{2} \cos \left(\lambda_{2} t\right)-\sqrt{2} C_{2} \sin \left(\lambda_{1} t\right)+\frac{P^{(0)}}{2} \cos \left(\lambda_{1} t\right), \\
& P_{-1,1}(t)= P_{-1,1}^{(0)} \cos \left(\lambda_{1} t\right)-\sqrt{2} C_{4} \sin \left(\lambda_{1} t\right), \\
& Q_{0,1}(t)= \frac{Q^{(0)}}{2}+C_{4} \cos \left(\lambda_{1} t\right)+\frac{\sqrt{2} P_{-1,1}^{(0)}}{2} \sin \left(\lambda_{1} t\right), \\
& Q_{-1,0}(t)= \frac{Q^{(0)}}{2}-C_{4} \cos \left(\lambda_{1} t\right)-\frac{\sqrt{2} P_{-1,1}^{(0)}}{2} \sin \left(\lambda_{1} t\right),
\end{aligned}
$$




$$
\begin{aligned}
& Q_{-1,1}(t)=Q_{-1,1}^{(0)}+\frac{1}{2 \sqrt{2}}\left[C_{3} \sin \left(\lambda_{2} t\right)+C_{5}\left(1-\cos \left(\lambda_{2} t\right)\right)\right], \\
& Q(t) \equiv Q^{(0)}, \quad P(t)=-2 \sqrt{2} C_{2} \sin \left(\lambda_{1} t\right)+P^{(0)} \cos \left(\lambda_{1} t\right), \quad t \geq 0
\end{aligned}
$$

with

$\lambda_{1}=\sqrt{2} B, \quad \lambda_{2}=2 \sqrt{2} B, \quad C_{1,2}=\frac{1}{2}\left(N_{1}^{(0)} \pm N_{-1}^{(0)}\right), \quad C_{3}=\frac{1}{2}\left(P_{0,1}^{(0)}-P_{-1,0}^{(0)}\right)$,

$C_{4}=\frac{1}{2}\left(Q_{0,1}^{(0)}-Q_{-1,0}^{(0)}\right), \quad C_{5}=\frac{1}{\sqrt{2}}\left[2-3\left(N_{1}^{(0)}+N_{-1}^{(0)}\right)-Q_{-1,1}^{(0)}\right]$.

These analytical solutions imply that: (a) $Q(t)$ is conserved; (b) if $B=0$, all the other quantities are also conserved; (c) if $B \neq 0$, they are periodic functions. The period of $N_{1}(t), N_{-1}(t), P_{0,1}(t), P_{-1,0}, P_{-1,1}(t), Q_{0,1}(t), Q_{-1,0}(t), M_{\Psi}(t)$ and $P(t)$ is $T=\sqrt{2} \pi /|B|$, while $N_{0}(t)$ and $Q_{-1,1}(t)$ have the same period $T=\pi /(\sqrt{2}|B|)$.

Proof. If $\beta_{s}=0$, noticing (2.19)-(2.23), the ODE system (2.7)-(2.15) collapses to

$$
\begin{aligned}
& N_{1}^{\prime}(t)-B P_{0,1}(t)=0, \\
& N_{0}^{\prime}(t)+B\left[P_{0,1}(t)-P_{-1,0}(t)\right]=0, \\
& N_{-1}^{\prime}(t)+B P_{-1,0}(t)=0 \\
& P_{0,1}^{\prime}(t)-2 B\left[N_{0}(t)-N_{1}(t)\right]+B Q_{-1,1}(t)-\left(E_{1}-E_{0}\right) Q_{0,1}(t)=0, \\
& P_{-1,0}^{\prime}(t)+2 B\left[N_{0}(t)-N_{-1}(t)\right]-B Q_{-1,1}(t)+\left(E_{-1}-E_{0}\right) Q_{-1,0}(t)=0, \\
& P_{-1,1}^{\prime}(t)-B\left[Q_{-1,0}(t)-Q_{0,1}(t)\right]-\left(E_{1}-E_{-1}\right) Q_{-1,1}(t)=0, \\
& Q_{0,1}^{\prime}(t)-B P_{-1,1}(t)+\left(E_{1}-E_{0}\right) P_{0,1}(t)=0, \\
& Q_{-1,0}^{\prime}(t)+B P_{-1,1}(t)-\left(E_{-1}-E_{0}\right) P_{-1,0}(t)=0, \\
& Q_{-1,1}^{\prime}(t)-B\left[P_{0,1}(t)-P_{-1,0}(t)\right]+\left(E_{1}-E_{-1}\right) P_{-1,1}(t)=0, \quad t \geq 0 .
\end{aligned}
$$

(i) If $B=0$, the above ODE system reduces to

$$
\begin{aligned}
& N_{1}^{\prime}(t)=0, \quad N_{0}^{\prime}(t)=0, \quad N_{-1}^{\prime}(t)=0, \quad t \geq 0 \\
& P_{0,1}^{\prime}(t)-\left(E_{1}-E_{0}\right) Q_{0,1}(t)=0, \quad Q_{0,1}^{\prime}(t)+\left(E_{1}-E_{0}\right) P_{0,1}(t)=0, \\
& P_{-1,0}^{\prime}(t)+\left(E_{-1}-E_{0}\right) Q_{-1,0}(t)=0, \quad Q_{-1,0}^{\prime}(t)-\left(E_{-1}-E_{0}\right) P_{-1,0}(t)=0 \\
& P_{-1,1}^{\prime}(t)-\left(E_{1}-E_{-1}\right) Q_{-1,1}(t)=0, \quad Q_{-1,1}^{\prime}(t)+\left(E_{1}-E_{-1}\right) P_{-1,1}(t)=0 .
\end{aligned}
$$

Thus (2.33) is the solution of the ODEs in (2.59) with initial data (2.16). With the initial condition (2.17) and (2.18), (2.34) and (2.35) is the solution of the ODEs in (2.60); (2.36) and (2.37) is the solution of the ODEs in (2.61), and (2.38) and (2.39) is the solution of the ODEs in (2.62). 
(ii) If $E_{1}=E_{0}=E_{-1}$, the ODE system (2.50)-(2.58) becomes

$$
\begin{aligned}
& N_{1}^{\prime}(t)-B P_{0,1}(t)=0 \\
& N_{0}^{\prime}(t)+B\left[P_{0,1}(t)-P_{-1,0}(t)\right]=0 \\
& N_{-1}^{\prime}(t)+B P_{-1,0}(t)=0 \\
& P_{0,1}^{\prime}(t)-2 B\left[N_{0}(t)-N_{1}(t)\right]+B Q_{-1,1}(t)=0, \\
& P_{-1,0}^{\prime}(t)+2 B\left[N_{0}(t)-N_{-1}(t)\right]-B Q_{-1,1}(t)=0, \\
& P_{-1,1}^{\prime}(t)-B\left[Q_{-1,0}(t)-Q_{0,1}(t)\right]=0, \\
& Q_{0,1}^{\prime}(t)-B P_{-1,1}(t)=0 \\
& Q_{-1,0}^{\prime}(t)+B P_{-1,1}(t)=0, \\
& Q_{-1,1}^{\prime}(t)-B\left[P_{0,1}(t)-P_{-1,0}(t)\right]=0, \quad t \geq 0
\end{aligned}
$$

Summing (2.69) and (2.70), we get

$$
Q^{\prime}(t)=Q_{0,1}^{\prime}(t)+Q_{-1,0}^{\prime}(t) \equiv 0, \quad t \geq 0 .
$$

This immediately implies that $Q(t)$ is conserved, i.e. the first part in (2.49). Subtracting (2.65) from (2.63), summing (2.66) and (2.67), we obtain

$$
\begin{aligned}
& N_{1}^{\prime}(t)-N_{-1}^{\prime}(t)-B\left[P_{0,1}(t)+P_{-1,0}(t)\right]=0, \\
& P_{0,1}^{\prime}(t)+P_{-1,0}^{\prime}(t)+2 B\left[N_{1}(t)-N_{-1}(t)\right]=0 .
\end{aligned}
$$

Solving the above linear ODE system and noticing the initial conditions (2.16) and (2.17), we have

$$
\begin{aligned}
& N_{1}(t)-N_{-1}(t)=2 C_{2} \cos \left(\lambda_{1} t\right)+\frac{1}{\sqrt{2}} P^{(0)} \sin \left(\lambda_{1} t\right), \\
& P(t)=P_{0,1}(t)+P_{-1,0}(t)=P^{(0)} \cos \left(\lambda_{1} t\right)-2 \sqrt{2} C_{2} \sin \left(\lambda_{1} t\right) .
\end{aligned}
$$

Summing (2.65) and (2.63), subtracting (2.67) from (2.66), noting (1.9), we have

$$
\begin{aligned}
& N_{1}^{\prime}(t)+N_{-1}^{\prime}(t)-B\left[P_{0,1}(t)-P_{-1,0}(t)\right]=0, \\
& P_{0,1}^{\prime}(t)-P_{-1,0}^{\prime}(t)+6 B\left[N_{1}(t)+N_{-1}(t)\right]+2 B Q_{-1,1}(t)=4 B .
\end{aligned}
$$

Solving the linear ODE system of (2.77), (2.78) and (2.71), noticing the initial conditions (2.16), (2.17) and (2.18), we get

$$
\begin{aligned}
& N_{1}(t)+N_{-1}(t)=2 C_{1}+\frac{1}{2 \sqrt{2}}\left[C_{3} \sin \left(\lambda_{2} t\right)+C_{5}\left(1-\cos \left(\lambda_{2} t\right)\right)\right], \\
& P_{0,1}(t)-P_{-1,0}(t)=C_{3} \cos \left(\lambda_{2} t\right)+C_{5} \sin \left(\lambda_{2} t\right), \\
& Q_{-1,1}(t)=Q_{-1,1}^{(0)}+\frac{1}{2 \sqrt{2}}\left[C_{3} \sin \left(\lambda_{2} t\right)+C_{5}\left(1-\cos \left(\lambda_{2} t\right)\right)\right] .
\end{aligned}
$$

Subtracting (2.70) from (2.69) leads to

$$
Q_{0,1}^{\prime}(t)-Q_{-1,0}^{\prime}(t)=2 B P_{-1,1}(t) .
$$

Solving (2.82) and (2.68) with the initial conditions (2.17) and (2.18), we get

$$
\begin{aligned}
& Q_{0,1}(t)-Q_{-1,0}(t)=2 C_{4} \cos \left(\lambda_{1} t\right)+\sqrt{2} P_{-1,1}^{(0)} \sin \left(\lambda_{1} t\right), \\
& P_{-1,1}(t)=P_{-1,1}^{(0)} \cos \left(\lambda_{1} t\right)-\sqrt{2} C_{4} \sin \left(\lambda_{1} t\right) .
\end{aligned}
$$

Thus we obtain the solution (2.40)-(2.49) from (2.75), (2.76), (2.79)-(2.81), (2.83), (2.84) and (1.9) immediately. 
3. Conservation of the angular momentum expectation. As a measure of vortex flux, we define the total angular momentum expectation

$$
\left\langle L_{z}\right\rangle(t)=\sum_{j=-1}^{1}\left\langle L_{z}\right\rangle_{j}(t), \quad t \geq 0
$$

where

$$
\begin{aligned}
& \left\langle L_{z}\right\rangle_{j}(t)=\int_{\mathbb{R}^{d}} \psi_{j}^{*} L_{z} \psi_{j} d \mathbf{x}=i \int_{\mathbb{R}^{d}} \psi_{j}^{*}\left(y \partial_{x}-x \partial_{y}\right) \psi_{j} d \mathbf{x}=-i \int_{\mathbb{R}^{d}} \psi_{j}^{*} \widehat{L}_{z} \psi_{j} d \mathbf{x}, \\
& \left\langle\widetilde{L_{z}}\right\rangle_{j}(t):=\frac{\left\langle L_{z}\right\rangle_{j}(t)}{N_{j}(t)}, \quad t \geq 0, \quad j=-1,0,1 .
\end{aligned}
$$

In fact, $\left\langle\widetilde{L_{z}}\right\rangle_{j}(t)$ is the angular momentum expectation of the $j$ th $(j=-1,0,1)$ component. As shown in Lemma 2.3, when $\beta_{s}=B=0$, the density of each component is conserved; thus in this case we have $\left\langle\widetilde{L_{z}}\right\rangle_{j}(t)=\frac{\left\langle L_{z}\right\rangle_{j}(t)}{N_{j}^{(0)}}$.

For the dynamics of the angular momentum expectation in rotating spin-1 BEC, we have the following lemmas:

Lemma 3.1. Suppose $\Psi(\mathbf{x}, t)$ is the solution of the CGPEs (1.1)-(1.4), then we have

$$
\frac{d\left\langle L_{z}\right\rangle(t)}{d t}=\left(\gamma_{y}^{2}-\gamma_{x}^{2}\right) \sum_{j=-1}^{1} \int_{\mathbb{R}^{d}} x y\left|\psi_{j}\right|^{2} d \mathbf{x}, \quad t \geq 0
$$

This suggests that, for any given initial data $\Psi^{0}(\mathbf{x})$, the total angular momentum expectation is conserved, i.e.

$$
\left\langle L_{z}\right\rangle(t)=\left\langle L_{z}\right\rangle(0)=\sum_{j=-1}^{1} \int_{\mathbb{R}^{d}}\left(\psi_{j}^{0}(\mathbf{x})\right)^{*} L_{z} \psi_{j}^{0}(\mathbf{x}) d \mathbf{x}, \quad t \geq 0
$$

if the external trapping potential $V(\mathbf{x})$ is radially symmetric in $2 D$, and resp. cylindrically symmetric in $3 D$, i.e. $\gamma_{x}=\gamma_{y}$ in (1.6).

Proof. Differentiating (3.2) with respect to $t$ for $j=1$, noticing (1.5) and (1.1), 
integrating by parts, we obtain

$$
\begin{aligned}
\frac{\left\langle L_{z}\right\rangle_{1}(t)}{d t}= & \int_{\mathbb{R}^{d}}\left[\partial_{t} \psi_{1}^{*} L_{z} \psi_{1}+\psi_{1}^{*} L_{z}\left(\partial_{t} \psi_{1}\right)\right] d \mathbf{x} \\
= & \int_{\mathbb{R}^{d}}\left[\left(-i \partial_{t} \psi_{1}^{*}\right) \widehat{L}_{z} \psi_{1}+\left(i \partial_{t} \psi_{1}\right) \widehat{L}_{z} \psi_{1}^{*}\right] d \mathbf{x} \\
= & \int_{\mathbb{R}_{d}}\left[-\frac{1}{2}\left(\widehat{L}_{z} \psi_{1} \nabla^{2} \psi_{1}^{*}+\widehat{L}_{z} \psi_{1}^{*} \nabla^{2} \psi_{1}\right)-i \Omega\left(\widehat{L}_{z} \psi_{1} \widehat{L}_{z} \psi_{1}^{*}-\widehat{L}_{z} \psi_{1}^{*} \widehat{L}_{z} \psi_{1}\right)\right. \\
& +\left(V(\mathbf{x})+E_{1}+\beta_{n} \rho+\beta_{s}\left(\rho_{1}+\rho_{0}-\rho_{-1}\right)\right)\left(\psi_{1}^{*} \widehat{L}_{z} \psi_{1}+\psi_{1} \widehat{L}_{z} \psi_{1}^{*}\right) \\
& \left.+\beta_{s}\left(\psi_{-1}\left(\psi_{0}^{*}\right)^{2} \widehat{L}_{z} \psi_{1}+\psi_{-1}^{*} \psi_{0}^{2} \widehat{L}_{z} \psi_{1}^{*}\right)+B\left(\psi_{0}^{*} \widehat{L}_{z} \psi_{1}+\psi_{0} \widehat{L}_{z} \psi_{1}^{*}\right)\right] d \mathbf{x} \\
= & \int_{\mathbb{R}^{d}}\left[\left(\gamma_{y}^{2}-\gamma_{x}^{2}\right) x y\left|\psi_{1}\right|^{2}+\left(\beta_{n}+\beta_{s}\right) \rho_{0} \widehat{L}_{z}\left|\psi_{1}\right|^{2}+\left(\beta_{n}-\beta_{s}\right) \rho_{-1} \widehat{L}_{z}\left|\psi_{1}\right|^{2}\right. \\
& \left.+\beta_{s}\left(\psi_{-1}\left(\psi_{0}^{*}\right)^{2} \widehat{L}_{z} \psi_{1}+\psi_{-1}^{*} \psi_{0}^{2} \widehat{L}_{z} \psi_{1}^{*}\right)+B\left(\psi_{0}^{*} \widehat{L}_{z} \psi_{1}+\psi_{0} \widehat{L}_{z} \psi_{1}^{*}\right)\right] d \mathbf{x} \\
= & \left(\gamma_{y}^{2}-\gamma_{x}^{2}\right) \int_{\mathbb{R}^{d}} x y\left|\psi_{1}(\mathbf{x}, t)\right|^{2} d \mathbf{x}+G_{1}(t), \quad t \geq 0,
\end{aligned}
$$

where

$$
\begin{aligned}
G_{1}(t)= & \int_{\mathbb{R}^{d}}\left[\left(\beta_{n}+\beta_{s}\right) \rho_{0}+\left(\beta_{n}-\beta_{s}\right) \rho_{-1}\right] \widehat{L}_{z}\left|\psi_{1}\right|^{2} d \mathbf{x} \\
& +2 \operatorname{Re} \int_{\mathbb{R}^{d}}\left[\beta_{s} \psi_{-1}\left(\psi_{0}^{*}\right)^{2} \widehat{L}_{z} \psi_{1}+B \psi_{0}^{*} \widehat{L}_{z} \psi_{1}\right] d \mathbf{x}, \quad t \geq 0
\end{aligned}
$$

Similarly, we have

$$
\begin{aligned}
& \frac{\left\langle L_{z}\right\rangle_{0}(t)}{d t}=\left(\gamma_{y}^{2}-\gamma_{x}^{2}\right) \int_{\mathbb{R}^{d}} x y\left|\psi_{0}(\mathbf{x}, t)\right|^{2} d \mathbf{x}+G_{0}(t) \\
& \frac{\left\langle L_{z}\right\rangle_{-1}(t)}{d t}=\left(\gamma_{y}^{2}-\gamma_{x}^{2}\right) \int_{\mathbb{R}^{d}} x y\left|\psi_{-1}(\mathbf{x}, t)\right|^{2} d \mathbf{x}+G_{-1}(t), \quad t \geq 0
\end{aligned}
$$

with

$$
\begin{aligned}
G_{0}(t)= & \left(\beta_{n}+\beta_{s}\right) \int_{\mathbb{R}_{d}}\left(\rho_{1}+\rho_{-1}\right) \widehat{L}_{z}\left|\psi_{0}\right|^{2} d \mathbf{x} \\
& +2 \operatorname{Re} \int_{\mathbb{R}_{d}}\left[\beta_{s} \psi_{-1}^{*} \psi_{1}^{*} \widehat{L}_{z} \psi_{0}^{2}+B\left(\psi_{1}^{*}+\psi_{-1}^{*}\right) \widehat{L}_{z} \psi_{0}\right] d \mathbf{x}, \\
G_{-1}(t)= & \int_{\mathbb{R}^{d}}\left[\left(\beta_{n}+\beta_{s}\right) \rho_{0}+\left(\beta_{n}-\beta_{s}\right) \rho_{1}\right] \widehat{L}_{z}\left|\psi_{-1}\right|^{2} d \mathbf{x} \\
& +2 \operatorname{Re} \int_{\mathbb{R}^{d}}\left[\beta_{s} \psi_{1}\left(\psi_{0}^{*}\right)^{2} \widehat{L}_{z} \psi_{-1}+B \psi_{0}^{*} \widehat{L}_{z} \psi_{-1}\right] d \mathbf{x}, \quad t \geq 0 .
\end{aligned}
$$

Summing (3.6), (3.8) and (3.9), noticing (3.7), (3.10) and (3.11), we get (3.4) immediately. $\square$

LEMMA 3.2. Suppose that the external trapping potential $V(\mathbf{x})$ is radially symmetric in 2D, and resp. cylindrically symmetric in 3D, i.e., $\gamma_{x}=\gamma_{y}$ in (1.6). The initial data $\psi_{j}^{0}(\mathbf{x})(j=-1,0,1)$ is chosen as

$$
\psi_{j}^{0}(\mathbf{x})=f_{j}(r) e^{i m_{j} \theta} \quad \text { with } m_{j} \in \mathbb{Z} \text { and } f_{j}(0)=0 \text { when } m_{j} \neq 0,
$$


in $2 D$, and resp. in $3 D$

$$
\psi_{j}^{0}(\mathbf{x})=f_{j}(r, z) e^{i m_{j} \theta} \quad \text { with } m_{j} \in \mathbb{Z} \text { and } f_{j}(0, z)=0 \text { when } m_{j} \neq 0 .
$$

If $\beta_{s}=B=0$, for any $\beta_{n}$ and $m_{j}(j=-1,0,1)$, the angular momentum expectation $\left\langle L_{z}\right\rangle_{j}(t)$ and $\left\langle\widetilde{L_{z}}\right\rangle_{j}(t)$ are conserved, i.e.

$$
\begin{aligned}
& \left\langle L_{z}\right\rangle_{j}(t) \equiv\left\langle L_{z}\right\rangle_{j}(0)=\int_{\mathbb{R}^{d}}\left(\psi_{j}^{0}(\mathbf{x})\right)^{*} L_{z} \psi_{j}^{0}(\mathbf{x}) d \mathbf{x}, \quad t \geq 0, \\
& \left\langle\widetilde{L_{z}}\right\rangle_{j}(t)=\left\langle\widetilde{L_{z}}\right\rangle_{j}(0)=\frac{1}{N_{j}^{(0)}} \int_{\mathbb{R}^{d}}\left(\psi_{j}^{0}(\mathbf{x})\right)^{*} L_{z} \psi_{j}^{0}(\mathbf{x}) d \mathbf{x}, \quad t \geq 0, \quad j=-1,0,1 .
\end{aligned}
$$

If $m_{-1}=m_{0}=m_{1}:=m$, for any $\beta_{n}, \beta_{s}$ and $B,\left\langle\widetilde{L_{z}}\right\rangle_{j}(t)$ is conserved, i.e.

$$
\left\langle\widetilde{L_{z}}\right\rangle_{j}(t) \equiv m, \quad t \geq 0, \quad j=-1,0,1
$$

Proof. If the initial data $\Psi^{0}(\mathbf{x})$ satisfies (3.12) in 2D, and resp. (3.13) in 3D, when $\beta_{s}=B=0$ or $m_{1}=m_{0}=m_{-1}:=m$, due to the symmetry, the solution $\Psi(\mathbf{x}, t)$ of the CGPEs (1.1)-(1.4) satisfies

$$
\psi_{j}(\mathbf{x}, t)=g_{j}(r, t) e^{i m_{j} \theta}, \quad \text { with } \quad g_{j}(r, 0)=f_{j}(r), \quad j=-1,0,1,
$$

in $2 \mathrm{D}$, and resp. in $3 \mathrm{D}$

$$
\psi_{j}(\mathbf{x}, t)=g_{j}(r, z, t) e^{i m_{j} \theta}, \quad \text { with } \quad g_{j}(r, z, 0)=f_{j}(r, z), \quad j=-1,0,1 .
$$

When $\beta_{s}=B=0$, plugging (3.17) for $2 \mathrm{D}$, or resp. (3.18) for $3 \mathrm{D}$, into (3.7), (3.10) and (3.11), we obtain

$$
G_{1}(t) \equiv 0, \quad G_{0}(t) \equiv 0, \quad G_{-1}(t) \equiv 0, \quad t \geq 0 .
$$

Substituting (3.19) into (3.6), (3.8) and (3.9), noticing $\gamma_{x}=\gamma_{y}$, we get

$$
\frac{\left\langle L_{z}\right\rangle_{1}(t)}{d t} \equiv 0, \quad \frac{\left\langle L_{z}\right\rangle_{0}(t)}{d t} \equiv 0, \quad \frac{\left\langle L_{z}\right\rangle_{-1}(t)}{d t} \equiv 0, \quad t \geq 0
$$

which implies the conservation of (3.14). The conservation in (3.15) is a combination of (3.14) and (2.2). When $m_{-1}=m_{0}=m_{1}:=m$, plugging (3.17) for 2D, or resp. (3.18) for $3 \mathrm{D}$, into (3.3), noticing (3.2) and (2.1), for $j=-1,0,1$, we obtain

$$
\begin{aligned}
\left\langle\widetilde{L_{z}}\right\rangle_{j}(t) & =\frac{\left\langle L_{z}\right\rangle_{j}(t)}{N_{j}(t)}=\frac{-i \int_{\mathbb{R}^{d}} \psi_{j}^{*} \widehat{L}_{z} \psi_{j} d \mathbf{x}}{\int_{\mathbb{R}^{d}}\left|\psi_{j}(\mathbf{x}, t)\right|^{2} d \mathbf{x}} \\
& =\frac{-i \int_{\mathbb{R}^{d}} g_{j}^{*} e^{-i m \theta}(i m) g_{j} e^{i m \theta} d \mathbf{x}}{\int_{\mathbb{R}^{d}}\left|g_{j}\right|^{2} d \mathbf{x}} \equiv m, \quad t \geq 0 .
\end{aligned}
$$

This immediately implies (3.16).

4. Dynamics of condensate widths. Another important quantity characterizing the dynamics of the spin-1 BEC is the condensate width defined as

$$
\sigma_{\alpha}(t)=\sqrt{\delta_{\alpha}(t)}=\sqrt{\delta_{\alpha, 1}(t)+\delta_{\alpha, 0}(t)+\delta_{\alpha,-1}(t)}, \quad \alpha=x, y, \text { or } z,
$$


where

$$
\delta_{\alpha, j}(t)=\left\langle\alpha^{2}\right\rangle_{j}(t)=\int_{\mathbb{R}^{d}} \alpha^{2}\left|\psi_{j}(\mathbf{x}, t)\right|^{2} d \mathbf{x}, \quad t \geq 0, \quad j=-1,0,1 .
$$

For the dynamics of condensate widths, we have the following lemmas.

Lemma 4.1. Suppose $\Psi(\mathbf{x}, t)$ is the solution of the CGPEs (1.1)-(1.4), then we have

$$
\begin{aligned}
\frac{d^{2} \delta_{\alpha}(t)}{d t^{2}}= & \int_{\mathbb{R}^{d}}\left[\sum _ { j = - 1 } ^ { 1 } 2 \left(\left(\partial_{y} \alpha-\partial_{x} \alpha\right)\left(2 i \Omega \psi_{j}^{*}\left(x \partial_{y}+y \partial_{x}\right) \psi_{j}+\Omega^{2}\left(x^{2}-y^{2}\right)\left|\psi_{j}\right|^{2}\right)\right.\right. \\
& \left.+\left|\partial_{\alpha} \psi_{j}\right|^{2}-\alpha\left|\psi_{j}\right|^{2} \partial_{\alpha}(V(\mathbf{x}))\right)+\beta_{n} \rho_{0}^{2}+\left(\beta_{n}+\beta_{s}\right)\left(\rho_{1}^{2}+\rho_{-1}^{2}+2 \rho_{0}\left(\rho_{1}+\rho_{-1}\right)\right) \\
& \left.+2\left(\beta_{n}-\beta_{s}\right) \rho_{1} \rho_{-1}+2 \beta_{s} \operatorname{Re}\left(\psi_{-1}^{*} \psi_{0}^{2} \psi_{1}^{*}\right)\right] d \mathbf{x}, \quad t \geq 0 \\
\delta_{\alpha}(0)= & \delta_{\alpha}^{(0)}=\int_{\mathbb{R}^{d}} \alpha^{2}\left(\left|\psi_{1}^{0}\right|^{2}+\left|\psi_{0}^{0}\right|^{2}+\left|\psi_{-1}^{0}\right|^{2}\right) d \mathbf{x}, \quad \alpha=x, y, z \\
\delta_{\alpha}^{\prime}(0)= & \delta_{\alpha}^{(1)}=2 \sum_{j=-1}^{1} \int_{\mathbb{R}^{d}} \alpha\left[-\Omega\left|\psi_{j}^{0}\right|^{2}\left(x \partial_{y} \alpha-y \partial_{x} \alpha\right)+\operatorname{Im}\left(\left(\psi_{j}^{0}\right)^{*} \partial_{\alpha} \psi_{j}^{0}\right)\right] d \mathbf{x}
\end{aligned}
$$

Proof. Differentiating (4.2) with respect to $t$ for $j=1$, noticing (1.1)-(1.3) and integrating by parts, we get

$$
\begin{aligned}
\frac{d \delta_{\alpha, 1}(t)}{d t}= & \frac{d}{d t} \int_{\mathbb{R}^{d}} \alpha^{2}\left|\psi_{1}(\mathbf{x}, t)\right|^{2} d \mathbf{x}=\int_{\mathbb{R}^{d}} \alpha^{2}\left(\psi_{1} \partial_{t} \psi_{1}^{*}+\psi_{1}^{*} \partial_{t} \psi_{1}\right) d \mathbf{x} \\
= & \int_{\mathbb{R}^{d}}\left[\frac{i}{2} \alpha^{2}\left(\psi_{1}^{*} \nabla^{2} \psi_{1}-\psi_{1} \nabla^{2} \psi_{1}^{*}\right)+i \beta_{s} \alpha^{2}\left(\psi_{-1}\left(\psi_{0}^{*}\right)^{2} \psi_{1}-\psi_{-1}^{*} \psi_{0}^{2} \psi_{1}^{*}\right)\right. \\
& \left.+\Omega \alpha^{2}\left(x \partial_{y}-y \partial_{x}\right)\left|\psi_{1}\right|^{2}+i B \alpha^{2}\left(\psi_{1} \psi_{0}^{*}-\psi_{1}^{*} \psi_{0}\right)\right] d \mathbf{x} \\
= & \int_{\mathbb{R}^{d}}\left[i \alpha\left(\psi_{1} \partial_{\alpha} \psi_{1}^{*}-\psi_{1}^{*} \partial_{\alpha} \psi_{1}\right)+i \beta_{s} \alpha^{2}\left(\psi_{-1}\left(\psi_{0}^{*}\right)^{2} \psi_{1}-\psi_{-1}^{*} \psi_{0}^{2} \psi_{1}^{*}\right)\right. \\
& \left.-2 \Omega \alpha\left|\psi_{1}\right|^{2}\left(x \partial_{y}-y \partial_{x}\right) \alpha+i B \alpha^{2}\left(\psi_{1} \psi_{0}^{*}-\psi_{1}^{*} \psi_{0}\right)\right] d \mathbf{x}
\end{aligned}
$$

Differentiating the above equation with respect to $t$, we obtain

$$
\begin{aligned}
\frac{d^{2} \delta_{\alpha, 1}(t)}{d t^{2}}= & \int_{\mathbb{R}^{d}}\left[2 i \alpha\left(\partial_{t} \psi_{1} \partial_{\alpha} \psi_{1}^{*}-\partial_{t} \psi_{1}^{*} \partial_{\alpha} \psi_{1}\right)-2 \Omega \alpha\left(\psi_{1} \partial_{t} \psi_{1}^{*}+\psi_{1}^{*} \partial_{t} \psi_{1}\right) \widehat{L}_{z} \alpha\right. \\
& +i\left(\psi_{1}^{*} \partial_{t} \psi_{1}-\psi_{1} \partial_{t} \psi_{1}^{*}\right)+i B \alpha^{2}\left(\psi_{0}^{*} \partial_{t} \psi_{1}+\psi_{1} \partial_{t} \psi_{0}^{*}-\psi_{0} \partial_{t} \psi_{1}^{*}-\psi_{1}^{*} \partial_{t} \psi_{0}\right) \\
& +i \beta_{s} \alpha^{2}\left(\psi_{-1}\left(\psi_{0}^{*}\right)^{2} \partial_{t} \psi_{1}+\psi_{1}\left(\psi_{0}^{*}\right)^{2} \partial_{t} \psi_{-1}+2 \psi_{-1} \psi_{0}^{*} \psi_{1} \partial_{t} \psi_{0}^{*}-\psi_{-1}^{*} \psi_{0}^{2} \partial_{t} \psi_{1}^{*}\right. \\
& \left.\left.-\psi_{1}^{*} \psi_{0}^{2} \partial_{t} \psi_{-1}^{*}-2 \psi_{-1}^{*} \psi_{0} \psi_{1}^{*} \partial_{t} \psi_{0}\right)\right] d \mathbf{x} \\
:= & I+I I+I I I+I V+V
\end{aligned}
$$


Plugging (1.1)-(1.3) into each parts of (4.7) and applying the integration by parts, we obtain

$$
\begin{aligned}
& I:=\int_{\mathbb{R}^{d}} 2 \alpha\left[\left(i \partial_{t} \psi_{1}\right) \partial_{\alpha} \psi_{1}^{*}+\left(-i \partial_{t} \psi_{1}^{*}\right) \partial_{\alpha} \psi_{1}\right] d \mathbf{x} \\
& =\int_{\mathbb{R}^{d}}\left[-\alpha\left(\partial_{\alpha} \psi_{1}^{*} \nabla^{2} \psi_{1}+\partial_{\alpha} \psi_{1} \nabla^{2} \psi_{1}^{*}\right)-2 \Omega \alpha\left(\partial_{\alpha} \psi_{1}^{*} L_{z} \psi_{1}+\partial_{\alpha} \psi_{1} L_{z}^{*} \psi_{1}^{*}\right)\right. \\
& +2 \alpha\left(V(\mathbf{x})+E_{1}+\beta_{n} \rho+\beta_{s}\left(\rho_{1}+\rho_{0}-\rho_{-1}\right)\right) \partial_{\alpha}\left|\psi_{1}\right|^{2} \\
& \left.+4 \alpha \operatorname{Re}\left(\beta_{s} \psi_{-1}^{*} \psi_{0}^{2} \partial_{\alpha} \psi_{1}^{*}+B \psi_{0} \partial_{\alpha} \psi_{1}^{*}\right)\right] d \mathbf{x} \\
& =2 \int_{\mathbb{R}^{d}}\left[-\frac{1}{2}\left|\nabla \psi_{1}\right|^{2}+\left|\partial_{\alpha} \psi_{1}\right|^{2}+\Omega \psi_{1}^{*} L_{z} \psi_{1}+i \Omega\left(\partial_{y} \alpha-\partial_{x} \alpha\right) \psi_{1}^{*}\left(x \partial_{y}+y \partial_{x}\right) \psi_{1}\right. \\
& -\left(V(\mathbf{x})+E_{1}+\left.\left.\left(\beta_{n}+\beta_{s}\right) \psi_{0}\right|^{2}\left|+\left(\beta_{n}-\beta_{s}\right)\right| \psi_{-1}\right|^{2}\right)\left|\psi_{1}\right|^{2} \\
& -\alpha\left|\psi_{1}\right|^{2} \partial_{\alpha}\left(V(\mathbf{x})+\left(\beta_{n}+\beta_{s}\right)\left|\psi_{0}\right|^{2}+\left(\beta_{n}-\beta_{s}\right)\left|\psi_{-1}\right|^{2}\right) \\
& \left.+2 \alpha \operatorname{Re}\left(\beta_{s} \psi_{-1}^{*} \psi_{0}^{2} \partial_{\alpha} \psi_{1}^{*}+B \psi_{0} \partial_{\alpha} \psi_{1}^{*}\right)\right] d \mathbf{x} \text {. } \\
& I I:=-2 \Omega \int_{\mathbb{R}^{d}} \alpha L_{z} \alpha\left[\psi_{1}^{*}\left(i \partial_{t} \psi_{1}\right)-\psi_{1}\left(-i \partial_{t} \psi_{1}^{*}\right)\right] d \mathbf{x} \\
& =-2 \Omega \int_{\mathbb{R}^{d}} \alpha L_{z} \alpha\left[-\frac{1}{2}\left(\psi_{1}^{*} \nabla^{2} \psi_{1}-\psi_{1} \nabla^{2} \psi_{1}^{*}\right)-\Omega\left(\psi_{1}^{*} L_{z} \psi_{1}-\psi_{1} L_{z}^{*} \psi_{1}^{*}\right)\right. \\
& \left.+\beta_{s}\left(\psi_{-1}^{*} \psi_{0}^{2} \psi_{1}^{*}-\psi_{-1}\left(\psi_{0}^{*}\right)^{2} \psi_{1}\right)+B\left(\psi_{1}^{*} \psi_{0}-\psi_{1} \psi_{0}^{*}\right)\right] d \mathbf{x} \\
& =2 \Omega \int_{\mathbb{R}^{d}}\left(\partial_{y} \alpha-\partial_{x} \alpha\right)\left[i \psi_{1}^{*}\left(x \partial_{y}+y \partial_{x}\right) \psi_{1}+\Omega\left(x^{2}-y^{2}\right)\left|\psi_{1}\right|^{2}\right. \\
& \left.+2 x y \operatorname{Im}\left(\beta_{s} \psi_{-1}^{*} \psi_{0}^{2} \psi_{1}^{*}+B \psi_{1}^{*} \psi_{0}\right)\right] d \mathbf{x} . \\
& I I I:=\int_{\mathbb{R}^{d}}\left[\psi_{1}^{*}\left(i \partial_{t} \psi_{1}\right)+\psi_{1}\left(-i \partial_{t} \psi_{1}^{*}\right)\right] d \mathbf{x} \\
& =\int_{\mathbb{R}^{d}}\left[-\frac{1}{2}\left(\psi_{1}^{*} \nabla^{2} \psi_{1}+\psi_{1} \nabla^{2} \psi_{1}^{*}\right)+2\left(V(\mathbf{x})+E_{1}\right)\left|\psi_{1}\right|^{2}-\Omega\left(\psi_{1}^{*} L_{z} \psi_{1}+\psi_{1} L_{z}^{*} \psi_{1}^{*}\right)\right. \\
& \left.+2\left(\beta_{n} \rho+\beta_{s}\left(\rho_{1}+\rho_{0}-\rho_{-1}\right)\right)\left|\psi_{1}\right|^{2}+2 \operatorname{Re}\left(\beta_{s} \psi_{-1}^{*} \psi_{0}^{2} \psi_{1}^{*}+B \psi_{1}^{*} \psi_{0}\right)\right] d \mathbf{x} \\
& =2 \int_{\mathbb{R}^{d}}\left[\frac{1}{2}\left|\nabla \psi_{1}\right|^{2}+\left(V(\mathbf{x})+E_{1}\right)\left|\psi_{1}\right|^{2}-\Omega \psi_{1}^{*} L_{z} \psi_{1}\right. \\
& \left.+\left(\beta_{n} \rho+\beta_{s}\left(\rho_{1}+\rho_{0}-\rho_{-1}\right)\right)\left|\psi_{1}\right|^{2}+\operatorname{Re}\left(\beta_{s} \psi_{-1}^{*} \psi_{0}^{2} \psi_{1}^{*}+B \psi_{1}^{*} \psi_{0}\right)\right] d \mathbf{x} . \\
& I V:=i B \int_{\mathbb{R}^{d}} \alpha^{2}\left(\psi_{0}^{*} \partial_{t} \psi_{1}+\psi_{1} \partial_{t} \psi_{0}^{*}-\psi_{0} \partial_{t} \psi_{1}^{*}-\psi_{1}^{*} \partial_{t} \psi_{0}\right) d \mathbf{x} . \\
& V:=i \beta_{s} \int_{\mathbb{R}^{d}} \alpha^{2}\left(\psi_{-1}\left(\psi_{0}^{*}\right)^{2} \partial_{t} \psi_{1}+\psi_{1}\left(\psi_{0}^{*}\right)^{2} \partial_{t} \psi_{-1}+2 \psi_{-1} \psi_{0}^{*} \psi_{1} \partial_{t} \psi_{0}^{*}\right. \\
& \left.-\psi_{-1}^{*} \psi_{0}^{2} \partial_{t} \psi_{1}^{*}-\psi_{1}^{*} \psi_{0}^{2} \partial_{t} \psi_{-1}^{*}-2 \psi_{-1}^{*} \psi_{0} \psi_{1}^{*} \partial_{t} \psi_{0}\right) d \mathbf{x} .
\end{aligned}
$$


Substituting (4.8)-(4.12) into (4.7), we obtain

$$
\begin{aligned}
\frac{d^{2} \delta_{\alpha, 1}(t)}{d t^{2}}=\int_{\mathbb{R}^{d}}[ & 2\left(\partial_{y} \alpha-\partial_{x} \alpha\right)\left(\Omega^{2}\left|\psi_{1}\right|^{2}\left(x^{2}-y^{2}\right)+2 i \Omega \psi_{1}^{*}\left(x \partial_{y}+y \partial_{x}\right) \psi_{1}\right) \\
& +2\left|\partial_{\alpha} \psi_{1}\right|^{2}+\left(\beta_{n}+\beta_{s}\right)\left|\psi_{1}\right|^{4}-2 \alpha\left|\psi_{1}\right|^{2} \partial_{\alpha}(V(\mathbf{x})) \\
& \left.-2 \alpha\left|\psi_{1}\right|^{2}\left(\left(\beta_{n}+\beta_{s}\right) \partial_{\alpha}\left|\psi_{0}\right|^{2}+\left(\beta_{n}-\beta_{s}\right) \partial_{\alpha}\left|\psi_{-1}\right|^{2}\right)\right] d \mathbf{x} \\
+ & \operatorname{Re} \int_{\mathbb{R}^{d}}\left[2 \beta_{s} \alpha \psi_{-1}^{*} \psi_{0}^{2} \partial_{\alpha} \psi_{1}^{*}+\beta_{s} \psi_{-1}^{*} \psi_{0}^{2} \psi_{1}^{*}+2 B \alpha \psi_{0} \partial_{\alpha} \psi_{1}^{*}+B \psi_{1}^{*} \psi_{0}\right] d \mathbf{x} \\
+2 & \operatorname{Im} \int_{\mathbb{R}^{d}}\left[2 \Omega x y\left(\partial_{y} \alpha-\partial_{x} \alpha\right)\left(\beta_{s} \psi_{-1}^{*} \psi_{0}^{2} \psi_{1}^{*}+B \psi_{1}^{*} \psi_{0}\right)\right. \\
& +\beta_{s} \alpha^{2}\left(\psi_{-1}^{*} \psi_{0}^{2} \partial_{t} \psi_{1}^{*}+\psi_{1}^{*} \psi_{0}^{2} \partial_{t} \psi_{-1}^{*}+2 \psi_{-1}^{*} \psi_{0} \psi_{1}^{*} \partial_{t} \psi_{0}\right) \\
& \left.+B \alpha^{2}\left(\psi_{0} \partial_{t} \psi_{1}^{*}+\psi_{1}^{*} \partial_{t} \psi_{0}\right)\right] d \mathbf{x} .
\end{aligned}
$$

Similarly, we get

$$
\begin{aligned}
& \frac{d^{2} \delta_{\alpha, 0}(t)}{d t^{2}}=\int_{\mathbb{R}^{d}}\left[2\left(\partial_{y} \alpha-\partial_{x} \alpha\right)\left(\Omega^{2}\left|\psi_{0}\right|^{2}\left(x^{2}-y^{2}\right)+2 i \Omega \psi_{0}^{*}\left(x \partial_{y}+y \partial_{x}\right) \psi_{0}\right)\right. \\
& +2\left|\partial_{\alpha} \psi_{0}\right|^{2}+\beta_{n}\left|\psi_{0}\right|^{4}-2 \alpha\left|\psi_{0}\right|^{2} \partial_{\alpha}(V(\mathbf{x})) \\
& \left.-2 \alpha\left(\beta_{n}+\beta_{s}\right)\left|\psi_{0}\right|^{2}\left(\partial_{\alpha}\left|\psi_{1}\right|^{2}+\partial_{\alpha}\left|\psi_{-1}\right|^{2}\right)\right] d \mathbf{x} \\
& +2 \operatorname{Re} \int_{\mathbb{R}^{d}}\left[2 \beta_{s}\left(2 \alpha \psi_{-1} \psi_{0}^{*} \psi_{1} \partial_{\alpha} \psi_{0}^{*}+\psi_{-1}\left(\psi_{0}^{*}\right)^{2} \psi_{1}\right)\right. \\
& \left.+B\left(\psi_{1}+\psi_{-1}\right)\left(\psi_{0}^{*}+2 \alpha \partial_{\alpha} \psi_{0}^{*}\right)\right] d \mathbf{x} \\
& +2 \operatorname{Im} \int_{\mathbb{R}^{d}}\left[2 \Omega x y\left(\partial_{y} \alpha-\partial_{x} \alpha\right)\left(2 \beta_{s} \psi_{-1}\left(\psi_{0}^{*}\right)^{2} \psi_{1}+B \psi_{0}^{*}\left(\psi_{1}+\psi_{-1}\right)\right)\right. \\
& +2 \beta_{s} \alpha^{2}\left(\left(\psi_{0}^{*}\right)^{2} \psi_{1} \partial_{t} \psi_{-1}+\psi_{-1}\left(\psi_{0}^{*}\right)^{2} \partial_{t} \psi_{1}+2 \psi_{-1} \psi_{0}^{*} \psi_{1} \partial_{t} \psi_{0}^{*}\right) \\
& \left.+B \alpha^{2}\left(\left(\psi_{1}+\psi_{-1}\right) \partial_{t} \psi_{0}^{*}+\psi_{0}^{*}\left(\partial_{t} \psi_{1}+\partial_{t} \psi_{-1}\right)\right)\right] d \mathbf{x} . \\
& \frac{d^{2} \delta_{\alpha,-1}(t)}{d t^{2}}=\int_{\mathbb{R}^{d}}\left[2\left(\partial_{y} \alpha-\partial_{x} \alpha\right)\left(\Omega^{2}\left|\psi_{-1}\right|^{2}\left(x^{2}-y^{2}\right)+2 i \Omega \psi_{-1}^{*}\left(x \partial_{y}+y \partial_{x}\right) \psi_{-1}\right)\right. \\
& +2\left|\partial_{\alpha} \psi_{-1}\right|^{2}+\left(\beta_{n}+\beta_{s}\right)\left|\psi_{-1}\right|^{4}-2 \alpha\left|\psi_{-1}\right|^{2} \partial_{\alpha}(V(\mathbf{x})) \\
& \left.-2 \alpha\left|\psi_{-1}\right|^{2}\left(\left(\beta_{n}+\beta_{s}\right) \partial_{\alpha}\left|\psi_{0}\right|^{2}+\left(\beta_{n}-\beta_{s}\right) \partial_{\alpha}\left|\psi_{1}\right|^{2}\right)\right] d \mathbf{x} \\
& +2 \operatorname{Re} \int_{\mathbb{R}^{d}}\left(2 \beta_{s} \alpha \psi_{1}^{*} \psi_{0}^{2} \partial_{\alpha} \psi_{-1}^{*}+\beta_{s} \psi_{1}^{*} \psi_{0}^{2} \psi_{-1}^{*}+2 B \alpha \psi_{0} \partial_{\alpha} \psi_{-1}^{*}+B \psi_{-1}^{*} \psi_{0}\right) d \mathbf{x} \\
& +2 \operatorname{Im} \int_{\mathbb{R}^{d}}\left[2 \Omega x y\left(\partial_{y} \alpha-\partial_{x} \alpha\right)\left(\beta_{s} \psi_{1}^{*} \psi_{0}^{2} \psi_{-1}^{*}+B \psi_{-1}^{*} \psi_{0}\right)\right. \\
& +\beta_{s} \alpha^{2}\left(\psi_{1}^{*} \psi_{0}^{2} \partial_{t} \psi_{-1}^{*}+\psi_{-1}^{*} \psi_{0}^{2} \partial_{t} \psi_{1}^{*}+2 \psi_{-1}^{*} \psi_{0} \psi_{1}^{*} \partial_{t} \psi_{0}\right) \\
& \left.+B \alpha^{2}\left(\psi_{0} \partial_{t} \psi_{-1}^{*}+\psi_{-1}^{*} \partial_{t} \psi_{0}\right)\right] d \mathbf{x} \text {. }
\end{aligned}
$$


Summing (4.13), (4.14) and (4.15), we obtain (4.3) immediately. In addition, the initial conditions (4.4) and (4.5) can be obtained from (4.1), (4.2) and (4.6) by setting $t=0$ and noticing (1.4).

Lemma 4.2. Suppose $\Psi(\mathbf{x}, t)$ is the solution of the CGPEs (1.1)-(1.4) with $B=0$ and $E_{1}=E_{0}=E_{-1}:=E$, then we have

(i) In $1 D$ without nonlinear term, i.e. $d=1, \beta_{n}=\beta_{s}=0$ and $\Omega=0$ in (1.1)-(1.3), for any initial data $\Psi^{0}=\Psi^{0}(x)$ in (1.4), we have

$$
\delta_{x}(t)=\frac{E_{\Psi}(0)-E}{\gamma_{x}^{2}}\left[1-\cos \left(2 \gamma_{x} t\right)\right]+\delta_{x}^{(0)} \cos \left(2 \gamma_{x} t\right)+\frac{\delta_{x}^{(1)}}{2 \gamma_{x}} \sin \left(2 \gamma_{x} t\right), \quad t \geq 0 .
$$

(ii) In $2 D$ with a radially symmetric trap, i.e. $d=2$ and $\gamma_{x}=\gamma_{y}:=\gamma_{r}$ in (1.1)-(1.3), for any initial data $\Psi^{0}=\Psi^{0}(x, y)$ in (1.4), we have, for time $t \geq 0$,

$$
\delta_{r}(t)=\frac{E_{\Psi}(0)+\Omega\left\langle L_{z}\right\rangle(0)-E}{\gamma_{r}^{2}}\left[1-\cos \left(2 \gamma_{r} t\right)\right]+\delta_{r}^{(0)} \cos \left(2 \gamma_{r} t\right)+\frac{\delta_{r}^{(1)}}{2 \gamma_{r}} \sin \left(2 \gamma_{r} t\right),
$$

where $\delta_{r}(t)=\delta_{x}(t)+\delta_{y}(t), \delta_{r}^{(0)}:=\delta_{x}(0)+\delta_{y}(0)$ and $\delta_{r}^{(1)}:=\delta_{x}^{\prime}(0)+\delta_{y}^{\prime}(0)$.

Proof. (i) When $\beta_{n}=\beta_{s}=0, B=0, \Omega=0$ and $E_{1}=E_{0}=E_{-1}:=E$ in (1.1)-(1.3), from (4.3) with $d=1$, noticing (1.10), we have

$$
\begin{aligned}
\frac{d^{2} \delta_{x}(t)}{d t^{2}} & =-2 \gamma_{x}^{2} \delta_{x}(t)+\int_{\mathbb{R}^{d}} \sum_{j=-1}^{1} 2\left|\partial_{x} \psi_{j}\right|^{2} \\
& =-4 \gamma_{x}^{2} \delta_{x}(t)+4 \int_{\mathbb{R}^{d}} \sum_{j=-1}^{1}\left(\frac{1}{2}\left|\partial_{x} \psi_{j}\right|^{2}+(V(x)+E)\left|\psi_{j}\right|^{2}\right)-4 E \\
& =-4 \gamma_{x}^{2} \delta_{x}(t)+4 E_{\Psi}(0)-4 E, \quad t \geq 0 .
\end{aligned}
$$

Thus, (4.16) is the unique solution of the second-order ODE (4.18) with the initial data (4.4) and (4.5).

(ii) Define

$$
\begin{aligned}
K(\Psi)= & \frac{\beta_{n}}{2} \rho_{0}^{2}+\frac{\beta_{n}+\beta_{s}}{2}\left(\rho_{1}^{2}+\rho_{-1}^{2}+2 \rho_{0}\left(\rho_{1}+\rho_{-1}\right)\right) \\
& +\left(\beta_{n}-\beta_{s}\right) \rho_{1} \rho_{-1}+\beta_{s} \operatorname{Re}\left(\psi_{-1}^{*} \psi_{0}^{2} \psi_{1}^{*}\right) .
\end{aligned}
$$

When $B=0, \gamma_{x}=\gamma_{y}:=\gamma_{r}$ and $E_{1}=E_{0}=E_{-1}:=E$ in (1.1)-(1.3), from (4.3) with $d=2$, we have

$$
\begin{aligned}
\frac{d^{2} \delta_{x}(t)}{d t^{2}}= & \int_{\mathbb{R}^{2}}\left[\sum _ { j = - 1 } ^ { 1 } 2 \left(\left|\partial_{x} \psi_{j}\right|^{2}-2 i \Omega \psi_{j}^{*}\left(x \partial_{y}+y \partial_{x}\right) \psi_{j}-\Omega^{2}\left(x^{2}-y^{2}\right)\left|\psi_{j}\right|^{2}\right.\right. \\
& \left.\left.-\gamma_{x}^{2} x^{2}\left|\psi_{j}\right|^{2}\right)+2 K(\Psi)\right] d \mathbf{x} \\
\frac{d^{2} \delta_{y}(t)}{d t^{2}}= & \int_{\mathbb{R}^{2}}\left[\sum _ { j = - 1 } ^ { 1 } 2 \left(\left|\partial_{y} \psi_{j}\right|^{2}+2 i \Omega \psi_{j}^{*}\left(x \partial_{y}+y \partial_{x}\right) \psi_{j}+\Omega^{2}\left(x^{2}-y^{2}\right)\left|\psi_{j}\right|^{2}\right.\right. \\
& \left.\left.-\gamma_{y}^{2} y^{2}\left|\psi_{j}\right|^{2}\right)+2 K(\Psi)\right] d \mathbf{x} .
\end{aligned}
$$


Summing (4.19) and (4.20), we obtain

$$
\begin{aligned}
\frac{d^{2} \delta_{r}(t)}{d t^{2}}= & -2 \gamma_{r}^{2} \delta_{r}(t)+\int_{\mathbb{R}^{2}}\left(\sum_{j=-1}^{1} 2\left|\nabla \psi_{j}\right|^{2}+4 K(\Psi)\right) d \mathbf{x} \\
= & -4 \gamma_{r}^{2} \delta_{r}(t)-4 E+4 \Omega\left\langle L_{z}\right\rangle(t) \\
& +4 \int_{\mathbb{R}^{2}} \sum_{j=-1}^{1}\left[\frac{1}{2}\left|\nabla \psi_{j}\right|^{2}+(V(\mathbf{x})+E)\left|\psi_{j}\right|^{2}-\Omega \operatorname{Re}\left(\psi_{j}^{*} L_{z} \psi_{j}\right)+K(\Psi)\right] d \mathbf{x} \\
= & -4 \gamma_{r}^{2} \delta_{r}(t)+4 E_{\Psi}(0)-4 E+4 \Omega\left\langle L_{z}\right\rangle(0), \quad t \geq 0 .
\end{aligned}
$$

Thus, (4.16) is the unique solution of the second-order ODE (4.21) with the initial data (4.4) and (4.5).

5. Dynamics of a stationary state with its center shifted. In this section, we assume that $B=0$ in (1.1)-(1.3). Let $\Phi:=\Phi(\mathbf{x})=\left(\phi_{1}(\mathbf{x}), \phi_{0}(\mathbf{x}), \phi_{-1}(\mathbf{x})\right)^{T}$ be a stationary state of the CGPEs (1.1)-(1.3), i.e.

$$
\begin{aligned}
\mu_{1} \phi_{1}(\mathbf{x})= & \left(-\frac{1}{2} \nabla^{2}+V(\mathbf{x})+E_{1}-\Omega L_{z}+\beta_{n} \rho+\beta_{s}\left(\rho_{1}+\rho_{0}-\rho_{-1}\right)\right) \phi_{1} \\
& +\beta_{s} \phi_{-1}^{*} \phi_{0}^{2}, \\
\mu_{0} \phi_{0}(\mathbf{x})= & \left(-\frac{1}{2} \nabla^{2}+V(\mathbf{x})+E_{0}-\Omega L_{z}+\beta_{n} \rho+\beta_{s}\left(\rho_{1}+\rho_{-1}\right)\right) \phi_{0}+2 \beta_{s} \phi_{-1} \phi_{0}^{*} \phi_{1}, \\
\mu_{-1} \phi_{-1}(\mathbf{x})= & \left(-\frac{1}{2} \nabla^{2}+V(\mathbf{x})+E_{-1}-\Omega L_{z}+\beta_{n} \rho+\beta_{s}\left(\rho_{-1}+\rho_{0}-\rho_{1}\right)\right) \phi_{-1} \\
& +\beta_{s} \phi_{1}^{*} \phi_{0}^{2},
\end{aligned}
$$

where $\mu_{1}, \mu_{0}$ and $\mu_{-1}$ are chemical potentials and they satisfy

$$
\mu_{1}+\mu_{-1}=2 \mu_{0} .
$$

If the initial data $\Psi^{0}(\mathbf{x})$ in $(1.4)$ is chosen as a stationary state with its center-of-mass shifted from the trap center, one can construct an exact solution of the CGPEs (1.1)(1.3) with a harmonic oscillator potential (1.6). This kind of analytical construction can be used, in particular, in the benchmark and validation of numerical algorithms for CGPEs. In [17, 11, 3, 36], similar kind of solutions have been constructed for the Gross-Pitaevskii equation of single-component BEC and CGPEs of two-component BEC. Here we extend this study to the spin-1 BEC.

Lemma 5.1. If the initial data $\Psi^{0}(\mathbf{x})$ in (1.4) is chosen as

$$
\Psi^{0}(\mathbf{x})=\Phi\left(\mathbf{x}-\mathbf{x}^{0}\right) e^{i\left(\mathbf{a}^{0} \cdot \mathbf{x}+b^{0}\right)}, \quad \mathbf{x} \in \mathbb{R}^{d},
$$

where $\mathbf{x}^{0}=\left(x_{1}^{0}, \ldots, x_{d}^{0}\right)^{T} \in \mathbb{R}^{d}$ is a given point, $\mathbf{a}^{0}=\left(a_{1}^{0}, \ldots, a_{d}^{0}\right)^{T} \in \mathbb{R}^{d}$ is a vector and $b^{0} \in \mathbb{R}$ is a constant, then the exact solution of (1.1)-(1.3) with the initial data (5.2) satisfies:

$$
\psi_{j}(\mathbf{x}, t)=\phi_{j}(\mathbf{x}-\mathbf{x}(t)) e^{-i \mu_{j} t} e^{i(\mathbf{a}(t) \cdot \mathbf{x}+b(t))}, \quad \mathbf{x} \in \mathbb{R}^{d}, \quad t \geq 0, \quad j=-1,0,1,
$$

where for any time $t \geq 0, \mathbf{x}(t)$ satisfies the following second-order ODE system:

$$
\begin{aligned}
& x^{\prime \prime}(t)-2 \Omega y^{\prime}(t)+\left(\gamma_{x}^{2}-\Omega^{2}\right) x(t)=0, \\
& y^{\prime \prime}(t)+2 \Omega x^{\prime}(t)+\left(\gamma_{y}^{2}-\Omega^{2}\right) y(t)=0, \quad t \geq 0, \\
& x(0)=x_{1}^{0}, \quad y(0)=x_{2}^{0}, \quad x^{\prime}(0)=\Omega x_{2}^{0}+a_{1}^{0}, \quad y^{\prime}(0)=-\Omega x_{1}^{0}+a_{2}^{0} .
\end{aligned}
$$


Moreover, if in 3D, another ODE needs to be added:

$$
z^{\prime \prime}(t)+\gamma_{z}^{2} z(t)=0, \quad z(0)=x_{3}^{0}, \quad z^{\prime}(0)=0 .
$$

In addition, $\mathbf{a}(t)=\left(a_{1}(t), \ldots, a_{d}(t)\right)^{T}$ and $b(t)$ satisfy

$$
\begin{aligned}
& \mathbf{a}^{\prime}(t)=-A \mathbf{x}(t)+\Omega J \mathbf{a}(t), \\
& b^{\prime}(t)=-\frac{3}{2}|\mathbf{a}(t)|^{2}-\frac{1}{2} \mathbf{x}(t)^{T} A \mathbf{x}(t)+\Omega \mathbf{x}(t)^{T} J \mathbf{a}(t), \quad t>0,
\end{aligned}
$$

with initial data

$$
\mathbf{a}(0)=\mathbf{a}^{0}, \quad b(0)=b^{0}
$$

where for $d=1$,

$$
A=\gamma_{x}^{2}, \quad J=0
$$

for $d=2$,

$$
A=\left(\begin{array}{cc}
\gamma_{x}^{2} & 0 \\
0 & \gamma_{y}^{2}
\end{array}\right), \quad J=\left(\begin{array}{cc}
0 & 1 \\
-1 & 0
\end{array}\right)
$$

for $d=3$,

$$
A=\left(\begin{array}{ccc}
\gamma_{x}^{2} & 0 & 0 \\
0 & \gamma_{y}^{2} & 0 \\
0 & 0 & \gamma_{z}^{2}
\end{array}\right), \quad J=\left(\begin{array}{ccc}
0 & 1 & 0 \\
-1 & 0 & 0 \\
0 & 0 & 0
\end{array}\right)
$$

Proof. The proof follows the line of the analogous result in [3] for GPE with an angular momentum rotation term for the single component BEC.

6. Numerical method and results. In this section, we present an efficient and accurate numerical method to discretize the CGPEs (1.1)-(1.3) for the dynamics of rotating spin-1 BEC and report some numerical results to validate our analytical results in previous sections.

6.1. Numerical method. In the literature, different spectrally accurate numerical methods were proposed to discretize GPE or CGPEs for studying the dynamics of BECs (cf. [7, 2, 3, 4] and references therein). The key ideas of these methods are: (i) using a time-splitting technique to decouple the nonlinearity; (ii) adopting the spectral method to discretize the free Schrödinger equation without/with the angular rotation term; (iii) constructing the proper spectral basis functions such that the reduced ODE system is decoupled and thus can be integrated in time exactly.

Here we will present a spectrally accurate method for the CGPEs (1.1)-(1.3) based on the above ideas. To do this, we first rewrite (1.1)-(1.3) into the form

$$
i \partial_{t} \Psi(\mathbf{x}, t)=C \Psi+D \Psi+G \Psi
$$

where the matrices

$$
C=\operatorname{diag}\{H, H, H\}, \quad D=\operatorname{diag}\left\{d_{1}, d_{2}, d_{3}\right\},
$$




$$
G=G(\Psi)=\left(\begin{array}{ccc}
0 & \beta_{s} \psi_{-1}^{*} \psi_{0}+B & 0 \\
\beta_{s} \psi_{-1} \psi_{0}^{*}+B & 0 & \beta_{s} \psi_{0}^{*} \psi_{1}+B \\
0 & \beta_{s} \psi_{0} \psi_{1}^{*}+B & 0
\end{array}\right)
$$

with

$H=-\frac{1}{2} \nabla^{2}-\Omega L_{z}, \quad d_{1}=V(\mathbf{x})+E_{1}+\beta_{n} \rho+\beta_{s}\left(\rho_{1}+\rho_{0}-\rho_{-1}\right)$,

$d_{2}=V(\mathbf{x})+E_{0}+\beta_{n} \rho+\beta_{s}\left(\rho_{1}+\rho_{-1}\right), \quad d_{3}=V(\mathbf{x})+E_{-1}+\beta_{n} \rho+\beta_{s}\left(\rho_{-1}+\rho_{0}-\rho_{1}\right)$.

Then the CGPEs (1.1)-(1.3) can be splitted into the following three subproblems:

$$
\begin{gathered}
i \partial_{t} \Psi(\mathbf{x}, t)=C \Psi(\mathbf{x}, t)=\left(-\frac{1}{2} \nabla^{2}-\Omega L_{z}\right) \Psi(\mathbf{x}, t), \\
i \partial_{t} \Psi(\mathbf{x}, t)=D \Psi(\mathbf{x}, t), \\
i \partial_{t} \Psi(\mathbf{x}, t)=G \Psi(\mathbf{x}, t) .
\end{gathered}
$$

For a given time step $\Delta t>0$, we denote the time sequence $t_{n}=n \Delta t$ for $n=0,1,2, \ldots$ Let $\Psi^{n}:=\Psi^{n}(\mathbf{x})=\left(\psi_{1}^{n}(\mathbf{x}), \psi_{0}^{n}(\mathbf{x}), \psi_{-1}^{n}(\mathbf{x})\right)^{T}$ be the approximation of $\Psi\left(\mathbf{x}, t_{n}\right)$. Then from time $t=t_{n}$ to $t=t_{n+1}$, a second-order symplectic time integrator for (6.1) can be given as follows $[32,4,8]$

$$
\begin{array}{lll}
\Psi^{(1)}=e^{-i C \Delta t / 2} \Psi^{n}, & \Psi^{(2)}=e^{-i D \Delta t / 2} \Psi^{(1)}, & \Psi^{(3)}=e^{-i G \Delta t} \Psi^{(2)}, \\
\Psi^{(4)}=e^{-i D \Delta t / 2} \Psi^{(3)}, & \Psi^{n+1}=e^{-i C \Delta t / 2} \Psi^{(4)} . &
\end{array}
$$

The key for an efficient implementation of (6.7) is to solve (6.4)-(6.6) efficiently on the time interval $\left[t_{n}, t_{n+1}\right]$. Different techniques were proposed in the literature for efficiently discretizing the subproblem (6.4) (cf. [3, 7, 37] and references therein). Here we adopt the technique proposed in $[3,37]$ for rotating BEC. The nonlinear ODE system (6.5) leaves $\left|\psi_{j}(\mathbf{x}, t)\right|(j=-1,0,1)$ invariant in time $t$, i.e., $\left|\psi_{j}(\mathbf{x}, t)\right|=$ $\left|\psi_{j}\left(\mathbf{x}, t_{n}\right)\right|$ for $t \in\left[t_{n}, t_{n+1}\right]$. Thus, the ODEs in (6.5) can be integrated exactly. For the nonlinear ODE system (6.6), we can not solve it exactly. Here, we use the approach applied in [8], which integrates (6.6) over the time interval $\left[t_{n}, t_{n+1}\right]$ and then approximates the integral by the second-order Runge-Kutta approximation [8]. The detailed scheme for (6.6) is

$$
\begin{aligned}
\Psi^{n+1} & \approx \Psi\left(\mathbf{x}, t_{n+1}\right)=\exp \left(-i \int_{t_{n}}^{t_{n+1}} G(\Psi(\mathbf{x}, \tau)) d \tau\right) \Psi\left(\mathbf{x}, t_{n}\right) \\
& \approx e^{-i \Delta t\left(G\left(\Psi^{n}\right)+G(\widetilde{\Psi})\right) / 2} \Psi^{n}:=e^{-i \Delta t R\left(\Psi^{n}\right)} \Psi^{n}
\end{aligned}
$$

where

$$
\begin{aligned}
& \widetilde{\Psi}=\Psi^{n}-i \Delta t G\left(\Psi^{n}\right) \Psi^{n}:=\left(\widetilde{\psi}_{1}, \widetilde{\psi}_{0}, \widetilde{\psi}_{-1}\right)^{T} \\
& R\left(\Psi^{n}\right)=\frac{1}{2}\left(G\left(\Psi^{n}\right)+G(\widetilde{\Psi})\right):=\left(\begin{array}{ccc}
0 & r_{12} & 0 \\
r_{12}^{*} & 0 & r_{23} \\
0 & r_{23}^{*} & 0
\end{array}\right)
\end{aligned}
$$

with

$$
r_{12}=\frac{\beta_{s}}{2}\left(\left(\psi_{-1}^{n}\right)^{*} \psi_{0}^{n}+\widetilde{\psi}_{-1}^{*} \widetilde{\psi}_{0}^{n}\right)+B, \quad r_{23}=\frac{\beta_{s}}{2}\left(\left(\psi_{0}^{n}\right)^{*} \psi_{1}^{n}+\widetilde{\psi}_{0}^{*} \widetilde{\psi}_{1}^{n}\right)+B .
$$


Since $G(\Psi)$ is a Hermitian matrix, $R\left(\Psi^{n}\right)$ is also a Hermitian matrix. Following [8], we can explicitly compute the approximation in (6.8) as

$$
\Psi^{n+1}=e^{-i \Delta t R\left(\Psi^{n}\right)} \Psi^{n}=\left(P e^{-i \Delta t \Lambda}\left(P^{*}\right)^{T}\right) \Psi^{n},
$$

where

$$
\Lambda=\left(\begin{array}{ccc}
0 & 0 & 0 \\
0 & \lambda & 0 \\
0 & 0 & -\lambda
\end{array}\right), \quad P=\frac{1}{\sqrt{2} \lambda}\left(\begin{array}{ccc}
\sqrt{2} r_{23} & r_{12} & -r_{12} \\
0 & \lambda & \lambda \\
-\sqrt{2} r_{12} & r_{23}^{*} & -r_{23}^{*}
\end{array}\right)
$$

with

$$
\lambda=\sqrt{\left|r_{12}\right|^{2}+\left|r_{23}\right|^{2}} .
$$

For more details, see $[8,4]$ and references therein.

6.2. Numerical results. In the following, we report some $2 \mathrm{D}$ numerical results to verify our analytical results about the dynamics of spin-1 BEC. The CGPEs (1.1)(1.3) is solved in the polar coordinate $(r, \theta)$ within a truncated domain $\Omega_{R}=\{\mathbf{x}|| \mathbf{x} \mid \leq$ $R$, where $R=20$ is large enough to neglect the truncation effect. We choose the mesh size $\Delta r=0.005$ and $\Delta \theta=\pi / 128$ and the time step $\Delta t=0.0001$.

EXAmple 1. Dynamics of the density of each component, we choose $d=2$, $\Omega=0.6, \gamma_{x}=\gamma_{y}=1.0, \beta_{n}=100$ in (1.1)-(1.3). The initial data in (1.4) is chosen as

$$
\psi_{1}^{0}(\mathbf{x})=(x+i y) \phi(\mathbf{x}), \quad \psi_{0}^{0}(\mathbf{x})=2 \phi(\mathbf{x}), \quad \psi_{-1}^{0}(\mathbf{x})=(x+2 i y) \phi(\mathbf{x})
$$

with

$$
\phi(\mathbf{x})=\frac{C}{\sqrt{\pi}} \exp \left(-\frac{x^{2}+y^{2}}{2}\right), \quad \mathbf{x} \in \mathbb{R}^{2},
$$

where the constant $C$ is chosen such that the initial data satisfies the normalization (1.9). Figure 1 shows time evolution of the density of each component and related quantities for four sets of parameters:

(i) $\beta_{s}=0, B=0, E_{1}=1, E_{0}=2, E_{-1}=4$;

(ii) $\beta_{s}=0, B=2, E_{1}=E_{0}=E_{-1}=1$;

(iii) $\beta_{s}=50, B=2, E_{1}=1, E_{0}=2, E_{-1}=3$;

(iv) $\beta_{s}=50, B=2, E_{1}=1, E_{0}=2, E_{-1}=4$.

From Fig. 1, we can draw the following conclusions: (i) the total density $N(t)$ is always conserved. (ii) When $\beta_{s}=0$, if furthermore $B=0$, then the density of each component is also conserved (cf. Fig. 1 for case (i)); otherwise, it evolves periodically if $B \neq 0$ (cf. Fig. 1 for case (ii)). In addition, if either $B=0$ or $E_{1}=E_{0}=E_{-1}$, the quantities $P_{l, k}(t)$ and $Q_{l, k}(t)$ are periodic functions (cf. Fig. 1 for cases (i)\&(ii)). The above results agree with those in Lemma 2.3. (iii) If $\beta_{s} \neq 0$ but $E_{1}+E_{-1}=2 E_{0}$, only $M_{\Psi}(t), P(t)$ and $Q(t)$ are periodic functions (cf. Fig. 1 for case (iii)), which confirms the results in Lemma 2.2 .

EXAMPLE 2. Dynamics of the angular momentum expectation, we choose $\Omega=$ $0.6, \beta_{n}=100, E_{1}=1, E_{0}=2$, and $E_{-1}=4$ in (1.1)-(1.3). The initial data in (1.4) is taken as

$$
\psi_{j}^{0}(\mathbf{x})=C r e^{-\frac{r^{2}}{2}} e^{i m_{j} \theta}, \quad j=-1,0,1,
$$



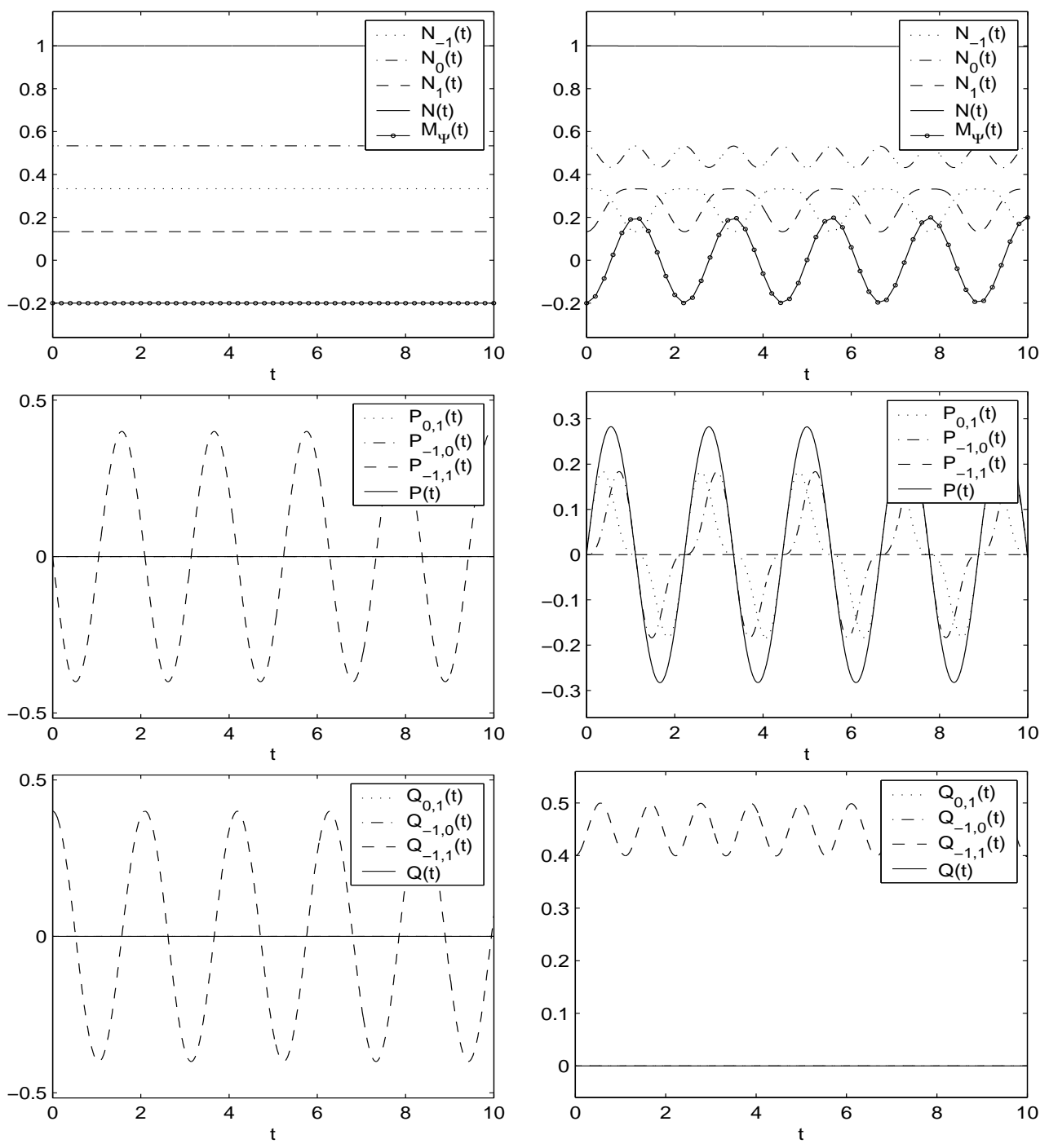
(right).

FIG. 1. Dynamics of $N_{j}(t), P_{l, k}(t)$ and $Q_{l, k}(t)$ in Example 1 for case (i) (left) and case (ii)

where the constant $C$ is chosen such that the initial data satisfies the normalization in (1.9). Figure 2 shows time evolution of the angular momentum expectation for four sets of parameters:

(i) $\gamma_{x}=\gamma_{y}=1, \beta_{s}=B=0, m_{1}=1, m_{0}=0$, and $m_{-1}=-1$;

(ii) $\gamma_{x}=\gamma_{y}=1, \beta_{s}=50, B=2, m_{1}=1, m_{0}=0$, and $m_{-1}=-1$;

(iii) $\gamma_{x}=\gamma_{y}=1, \beta_{s}=50, B=2, m_{j}=1(j=-1,0,1)$;

(iv) $\gamma_{x}=1, \gamma_{y}=2, \beta_{s}=50, B=2, m_{j}=1(j=-1,0,1)$.

Fig. 2 suggests that: (i) if $\gamma_{x}=\gamma_{y}$, the total angular momentum expectation $\left\langle L_{z}\right\rangle(t)$ is conserved for any time $t \geq 0$ (cf. Fig. 2a,b\&c). Furthermore, if $\beta_{s}=B=0$ or $m_{-1}=m_{0}=m_{1}:=m$, the angular momentum expectation for $j$-th component, i.e. $\left\langle\widetilde{L_{z}}\right\rangle_{j}(t)$, is also conserved for $j=-1,0,1$ (cf. Fig. 2a\&c). In addition, if $\beta_{s}=B=0$, 

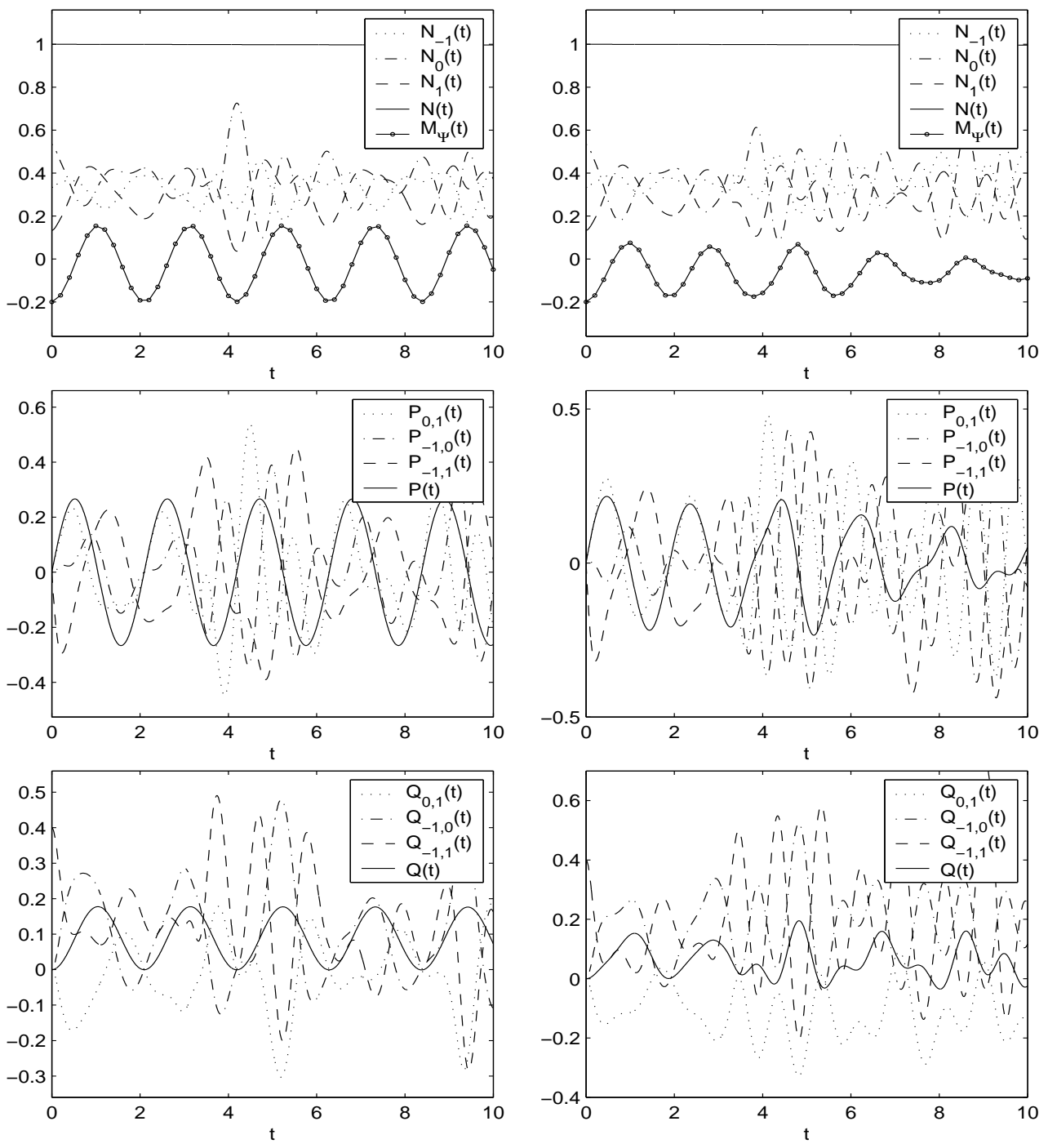

FIG. 1 (cont'd). For case (iii) (left) and case (iv) (right).

$\left\langle L_{z}\right\rangle_{j}(t)$ is also conserved for $j=-1,0,1$ (cf. Fig. 2a). (ii) If $\gamma_{x} \neq \gamma_{y}$, the angular momentum expectation $\left\langle L_{z}\right\rangle(t)$ and related quantities $\left\langle L_{z}\right\rangle_{j}(t)(j=-1,0,1)$ are, in general, not conserved (cf. Fig. 2d). These numerical results confirm the analytical results in Lemmas 3.1-3.2.

ExAmple 3. Dynamics of the condensate widths, we choose $d=2, \Omega=0.6$, $\beta_{n}=100, \beta_{s}=50, B=0$ and $E_{1}=E_{0}=E_{-1}=0$ in the CGPEs (1.1)-(1.3). The initial data is chosen as

$$
\psi_{1}^{0}(\mathbf{x})=C r e^{-\frac{r^{2}}{2}} e^{i \theta}, \quad \psi_{0}^{0}(\mathbf{x})=2 C r e^{-\frac{r^{2}}{2}}, \quad \psi_{-1}^{0}(\mathbf{x})=C r e^{-\frac{r^{2}}{2}} e^{-i \theta}
$$

with $C$ a constant ensuring that the initial data in (6.13) satisfy the normalization condition (1.9). Figure 3 shows time evolution of the condensate widths $\delta_{x}(t), \delta_{y}(t)$ and $\delta_{r}(t)$ for two sets of parameters: (i) $\gamma_{x}=\gamma_{y}=1$; (ii) $\gamma_{x}=1$ and $\gamma_{y}=2$. 
a)
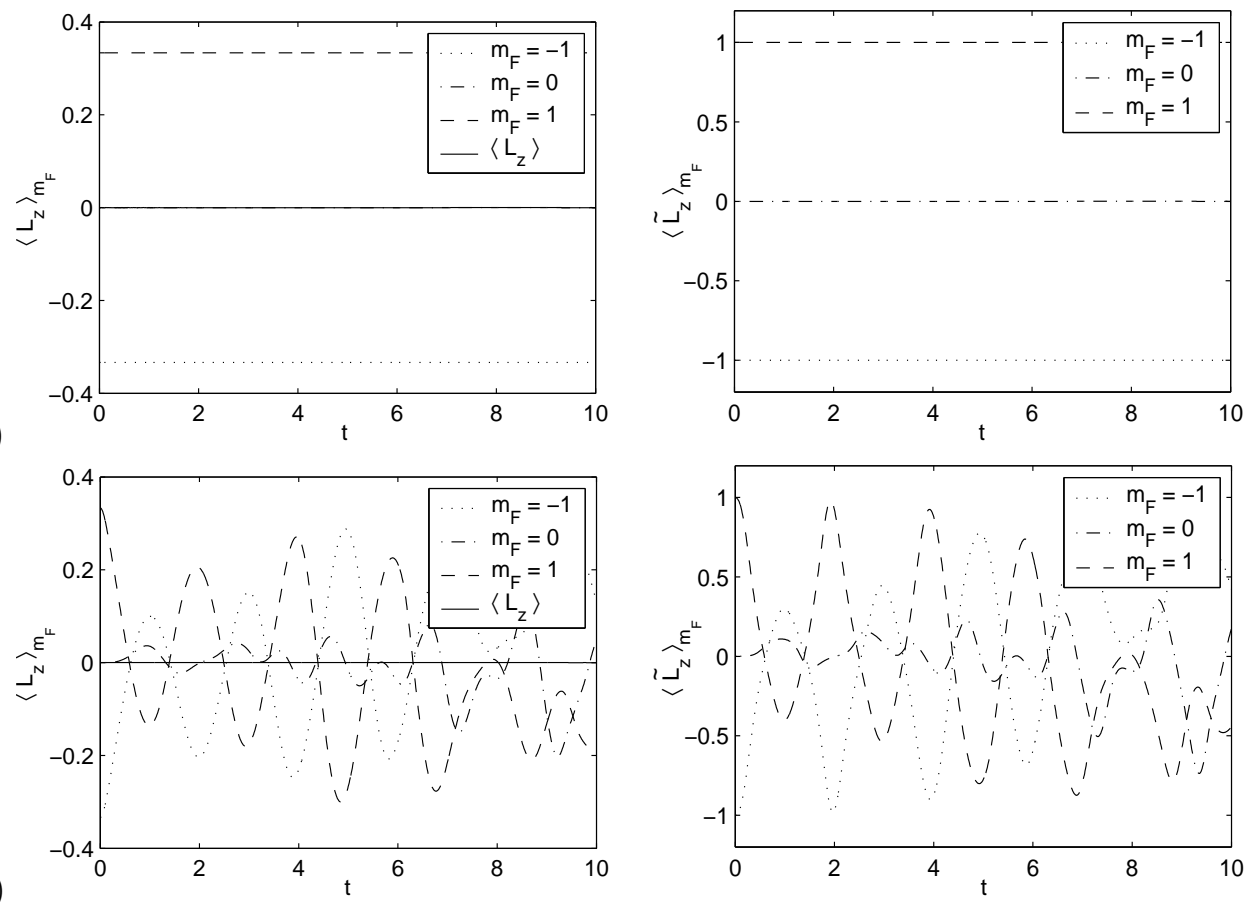

b)
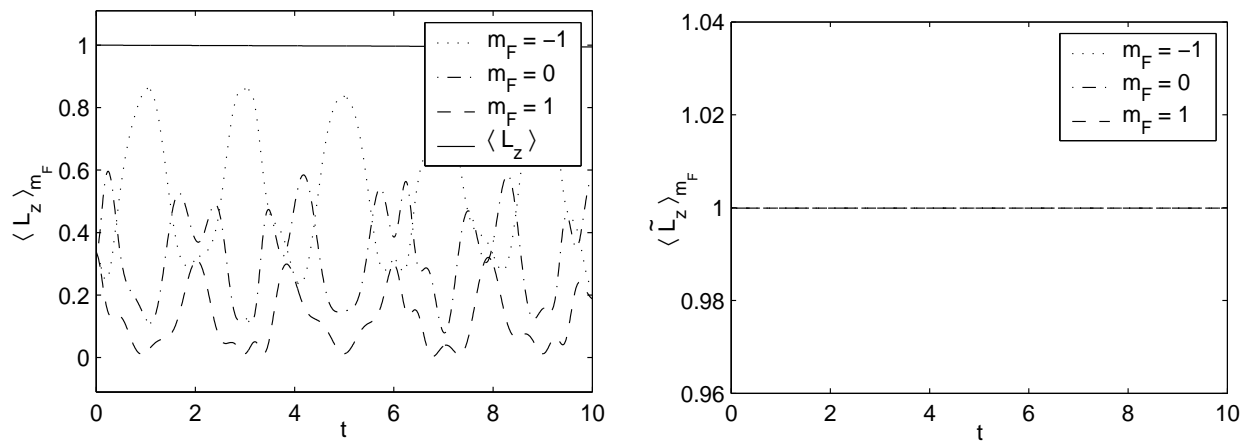

c)
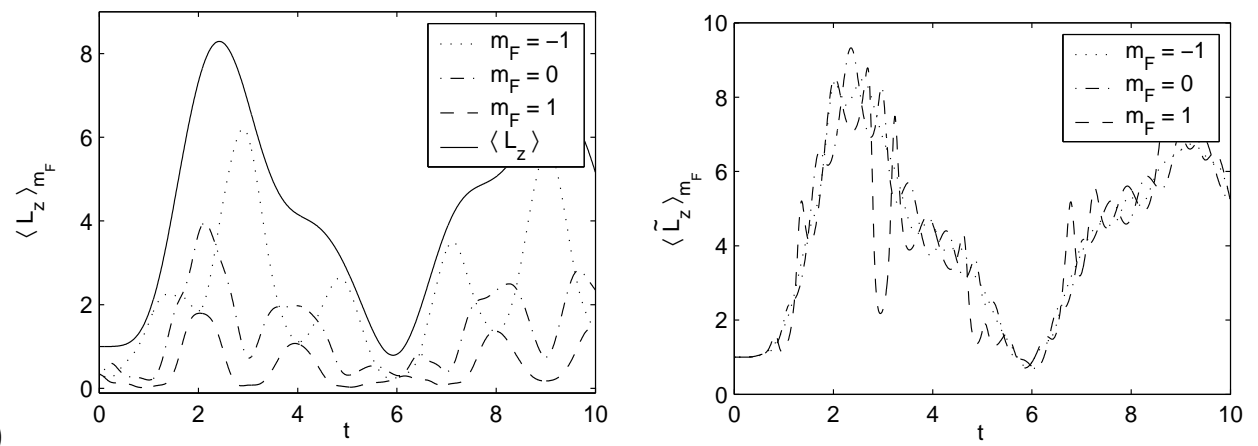

FIG. 2. Dynamics of the angular momentum expectation in Example 2 for a). case (i); b). case (ii); c). case (iii); d). case (iv).

Figure 3 shows that the condensate width $\delta_{r}(t)$ is a periodic function when $\gamma_{x}=$ $\gamma_{y}$. In addition, $\delta_{x}(t)=\delta_{y}(t)=\delta_{r}(t) / 2$ is also a periodic function (cf. Fig. 3a). 
a)

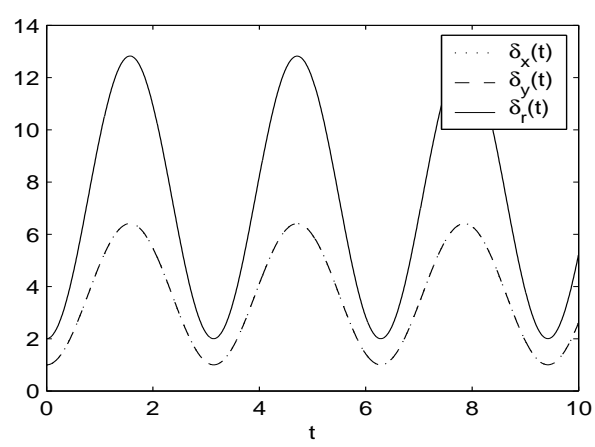

b)

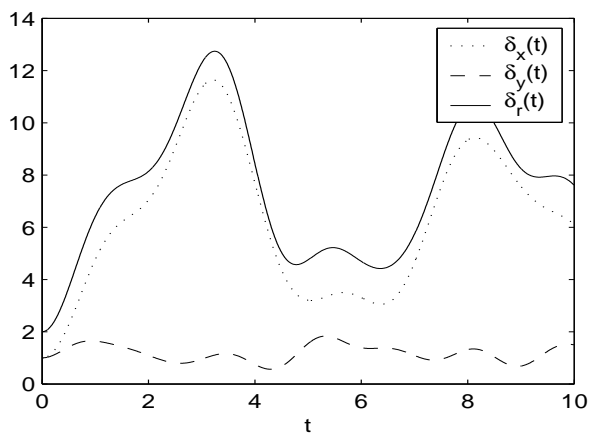

FIG. 3. Dynamics of the condensate widths in Example 3 for: a). case (i); b). case (ii).

While in general, they are not periodic when $\gamma_{x} \neq \gamma_{y}$ (cf. Fig. 3b).

In fact, the numerical results in the above three Examples not only confirm our analytically results obtained in previous sections but also demonstrate the accuracy of our numerical method for simulating the dynamics of spin-1 BEC.

EXAMPLE 4. Interaction of vortices in rotating spin-1 BEC, i.e., we take $d=2$, $\gamma_{x}=\gamma_{y}=1, B=0$ and $E_{1}=E_{0}=E_{-1}=0$ in (1.1)-(1.3). The initial data in (1.4) are chosen as follows:

Case I. Interaction of vortices with the same winding number, i.e.

$$
\psi_{1}^{0}(x, y)=\phi_{+}\left(x+x_{0}, y\right), \quad \psi_{0}^{0}(x, y)=2 \phi_{+}(x, y), \quad \psi_{-1}^{0}(x, y)=\phi_{+}\left(x-x_{0}, y\right) ;
$$

Case II. Interaction of vortices with opposite winding numbers, i.e.

$$
\psi_{1}^{0}(x, y)=\phi_{+}\left(x+x_{0}, y\right), \quad \psi_{0}^{0}(x, y)=2 \phi_{-}(x, y), \quad \psi_{-1}^{0}(x, y)=\phi_{+}\left(x-x_{0}, y\right) ;
$$

where

$$
\phi_{ \pm}(x, y)=\frac{C}{\sqrt{6 \pi}}(x \pm i y) e^{-\left(x^{2}+y^{2}\right) / 2}, \quad(x, y) \in \mathbb{R}^{2},
$$

represents a vortex state located at the origin with winding number \pm 1 , and $x_{0}$ is the initial position of the vortex. In our simulations, we choose $x_{0}=0.6$ in (6.14) and (6.15).

Figure 4 depicts time evolution of the vortex centers when the interaction parameters are small, i.e. $\beta_{n}=10$ and $\beta_{s}=5$, for different rotation speed $\Omega$. In fact, when $\beta_{n}$ and $\beta_{s}$ are small, the dynamics of the vortices in cases I and II are quite similar. On the other hand, the situation becomes more complicated when the interaction parameters $\beta_{n}$ and $\beta_{s}$ are large, especially in case II. Figures 5 and 6 show the densities $\rho_{j}(\mathbf{x}, t)=\left|\psi_{j}(\mathbf{x}, t)\right|^{2}(j=1,0,-1)$ and $\rho=\rho_{1}+\rho_{0}+\rho_{-1}$, and the phase $S_{j}$ $\left(\psi_{j}=\sqrt{\rho_{j}} e^{i S_{j}}, j=-1,0,1\right)$ at different times, respectively, with $\Omega=0.6, \beta_{n}=100$ and $\beta_{s}=90$, i.e. strong interaction, for case I. Figures 7 and 8 show similar results for case II. Figure 9 displays the dynamics of the angular momentum expectation with $\Omega=0.6, \beta_{n}=100$ and $\beta_{s}=90$ for cases I and II. In Fig. 4, the symbols '+' and ' - ' represent the initial location of a vortex with winding number +1 and -1 , respectively. While in Figs. 5-8, they represent the positions of vortex centers at a given time $t$. 

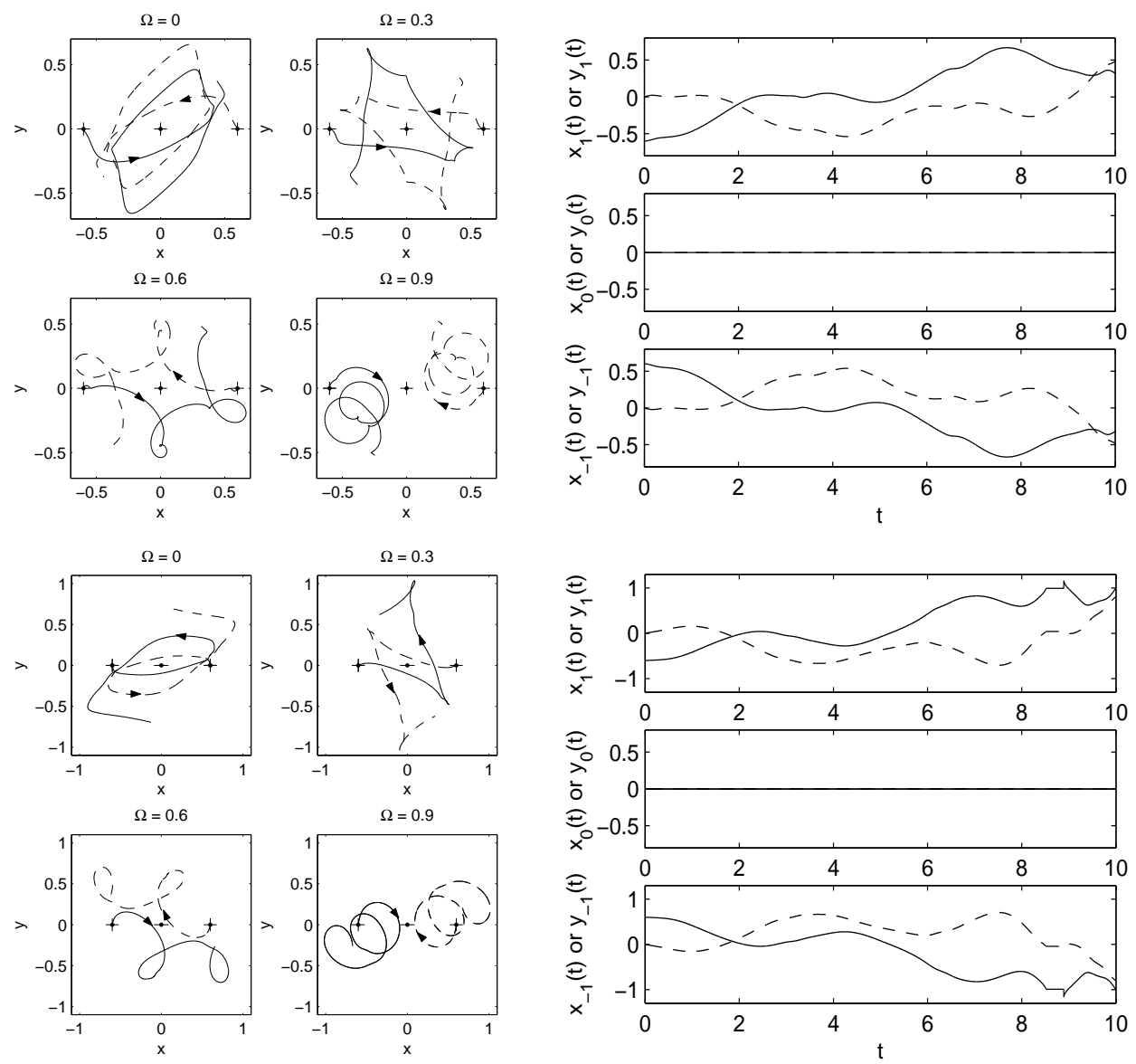

Fig. 4. Trajectories of vortex centers in Example 4 with $\beta_{n}=10$ and $\beta_{s}=5$, i.e. weak interaction, for different $\Omega$ (left) and time evolution of $x_{j}(t)$ and $y_{j}(t)$ (for $j=-1,0,1$ ) when $\Omega=0.6$ (right): a) for case I; and b) for case II.

From Fig. 4 and additional numerical results not shown here for brevity, we have the following observation for the vortex interaction in spin-1 BEC when the interaction parameters $\beta_{n}$ and $\beta_{s}$ are small: in both cases I and II, the vortex initially located at the origin of $m_{F}=0$ component does not move during the dynamics, while the other two vortices initially located at $( \pm 0.6,0)$ in spin component $m_{F}= \pm 1$ rotate around the origin (cf. Fig. 4); for different $\Omega$, the trajectories of the other two vortices located in $m_{F}= \pm 1$ components could be very different (cf. Fig. 4). In addition, we find that the dynamics of the vortices in cases I and II are quite similar. For both cases, the number of vortices is always conserved during our computational time, i.e. $t \in[0,10]$. On the other hand, from Figs. 5-9 and additional numerical results, we can draw the following conclusions when the interaction parameters $\beta_{n}$ and $\beta_{s}$ are large: (i) in case I, the vortex initially located at the origin in $m_{F}=0$ component does not move during the dynamics, while the other two vortices initially located at $( \pm 0.6,0)$ in spin component $m_{F}= \pm 1$ rotate around the origin (cf. Fig. 5). During the dynamics, these three vortices never collide and annihilate. (ii) In case II, the vortex initially located at the origin in spin component $m_{F}=0$ will disappear and then re-generate 


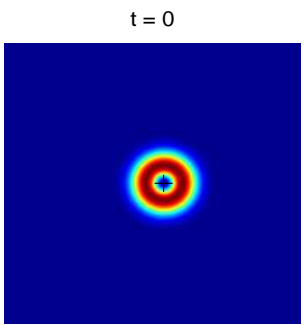

$t=1$

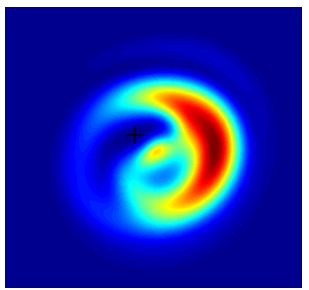

$t=5$

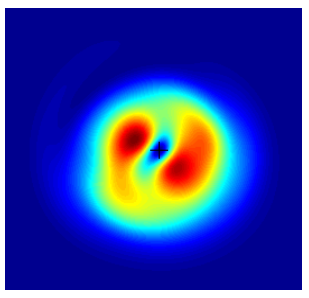

$t=10$

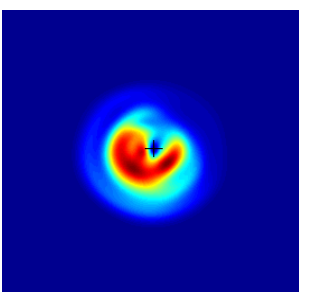

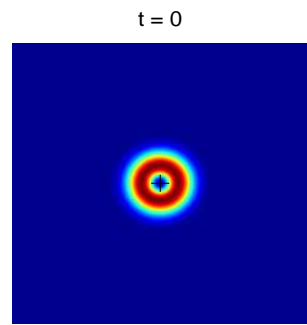

$t=1$

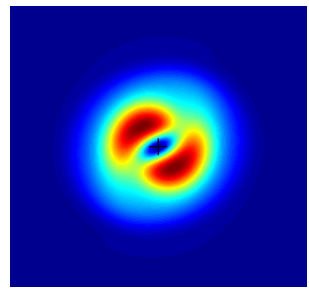

$t=5$

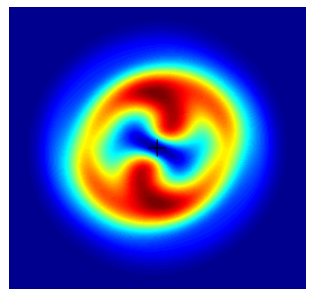

$t=10$

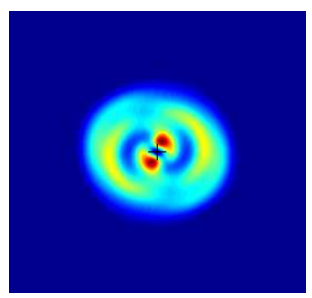

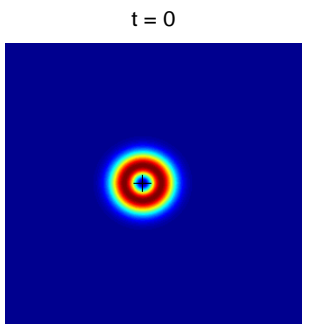

$t=1$

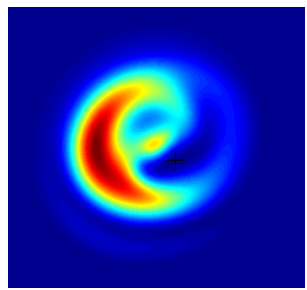

$t=5$

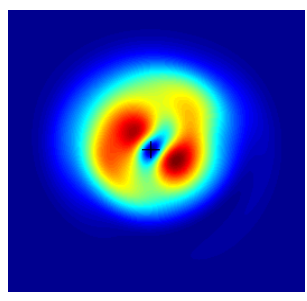

$t=10$

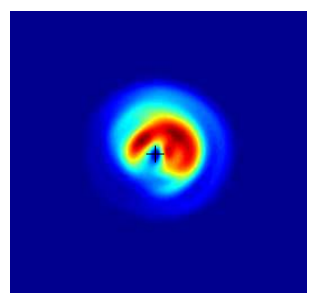

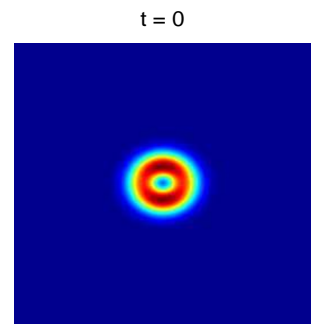

$t=1$

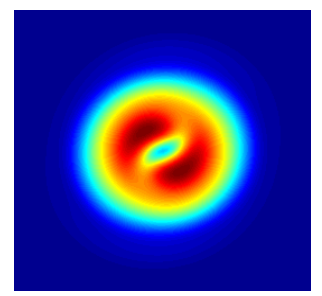

$t=5$

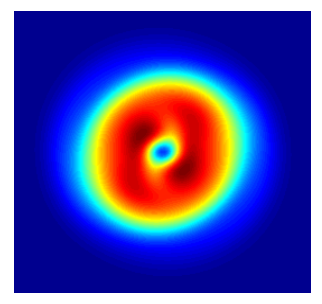

$t=10$

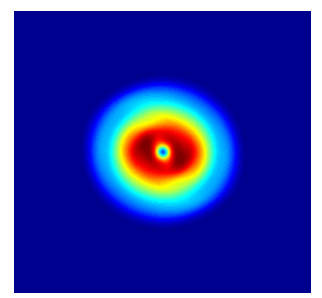

FIG. 5. Contour plots of the density $\rho_{-1}(t), \rho_{0}(t), \rho_{1}(t)$ and $\rho(t)$ (from left column to right column) over the dimensionless domain $[-8,8] \times[-8,8]$ in Example 4 with $\Omega=0.6, \beta_{n}=100$ and $\beta_{s}=90$ for case $I$.

after some time (cf. Fig. 8); the other two vortices initially located at $( \pm 0.6,0)$ in spin component $m_{F}= \pm 1$ rotate around the origin near $t=0$ (cf. Fig. 7). (iii) In case II, the number of the vortices is not conserved. During the dynamics, new vortices are generated near the boundary of each spin component and they propagate into the condensate and interact with other vortices in the same spin-component and different spin-components. (iv) In addition, our extensive numerical results show that the dynamics and interaction patterns highly depend on $x_{0}$ in (6.14) and (6.15). (v) The total angular momentum expectation $\left\langle L_{z}\right\rangle$ is conserved in cases I and II (cf. Fig. 9 ), which again confirms the analytical results (3.5). (vi) The motion of vortex centers in the interaction is affected by both angular momentum rotation speed $\Omega$ and the interaction strength $\beta_{n}$ and $\beta_{s}$ (cf. Figs. 4,6\&8). Similar to the single component case $[21,35]$, the above results show that the interaction of vortices in rotating spin-1 BEC might be very interesting and complicated, especially when $\beta_{n}$ and $\beta_{s}$ as well 


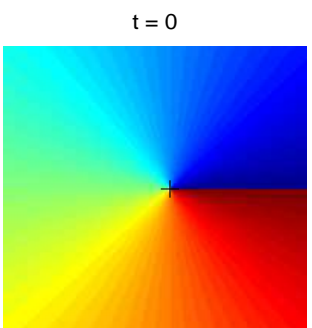

$t=1$

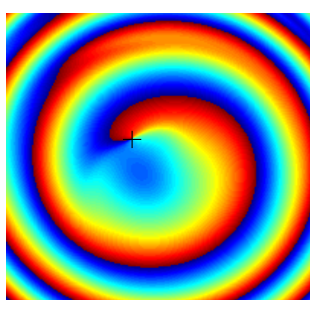

$t=5$

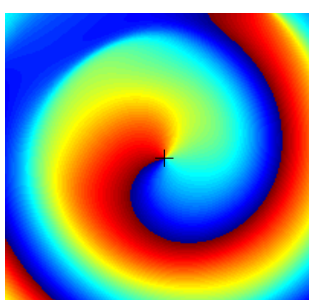

$t=10$

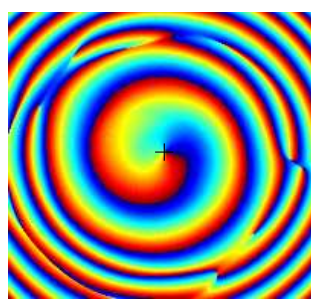

$t=0$

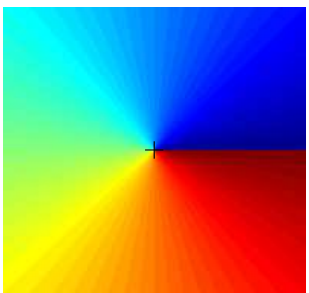

$t=1$

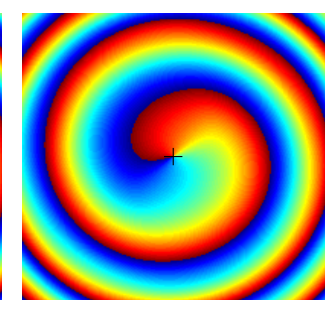

$t=5$

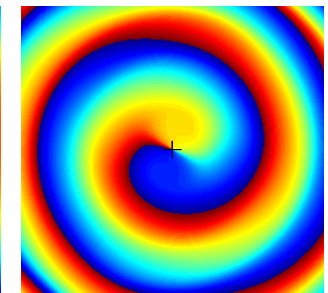

$t=10$ $t=0$

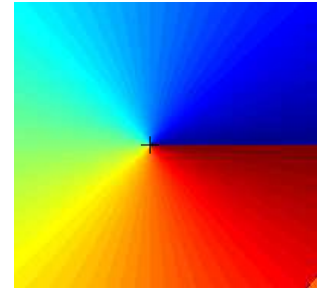

$t=1$

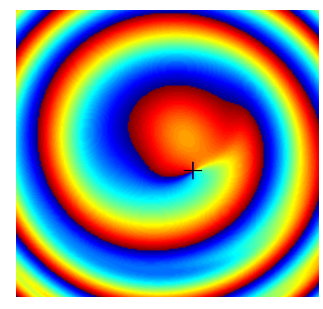

$t=5$

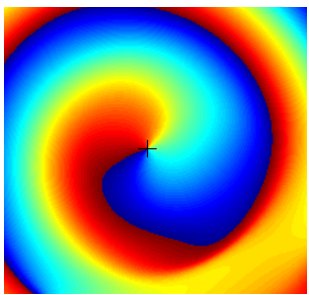

$t=10$
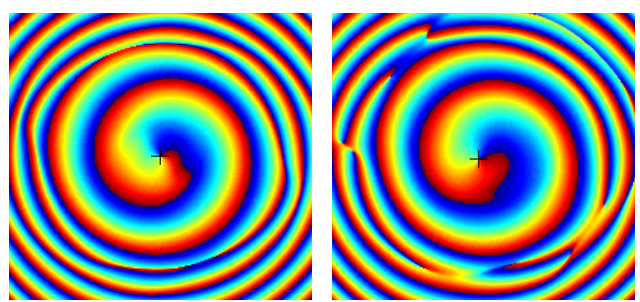

Fig. 6. Contour plots of the phase $S_{-1}(t), S_{0}(t)$ and $S_{1}(t)$ (from left column to right column) over the dimensionless domain $[-6,6] \times[-6,6]$ in Example 4 with $\Omega=0.6, \beta_{n}=100$ and $\beta_{s}=90$ for case $I$.

as $|\Omega|$ are large, and more systematic study will be carried out in our future study.

7. Concluding remarks. We have studied analytically and numerically the dynamical properties of the coupled Gross-Pitaevskii equations (CGPEs) without/with an angular momentum rotation term and an external magnetic field for the dynamics of nonrotating/rotating spin-1 Bose-Einstein condensates (BECs). Along the analytical front, we obtained a system of first order ordinary differential equations (ODEs) governing the dynamics of the density of each component and related quantities and solved the ODEs analytically in a few cases; proved the conservation of the angular momentum expectation when the external trapping potential is radially symmetric in two dimensions or cylindrically symmetric in three dimensions; derived a second order ODE for the dynamics of the condensate width and showed that it is a periodic function without/with a perturbation; and constructed the analytical solution of the 


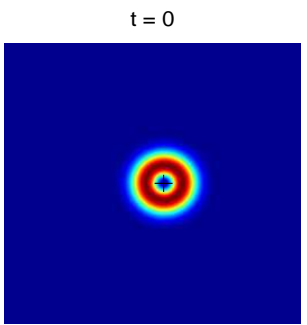

$t=1$

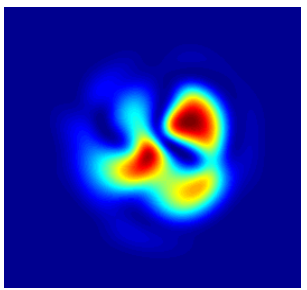

$t=5$

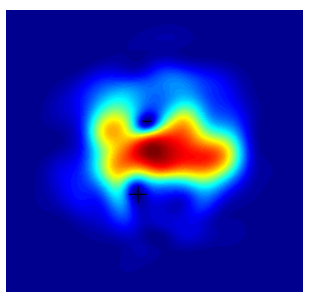

$\mathrm{t}=10$

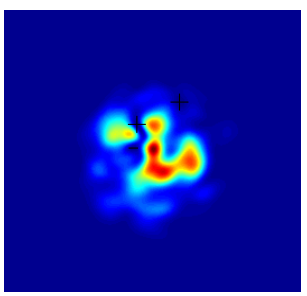

$t=0$

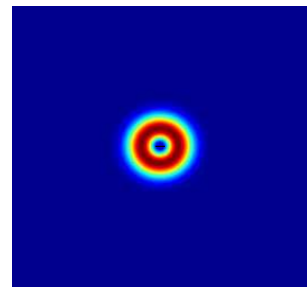

$t=1$

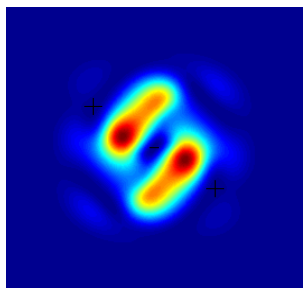

$t=5$

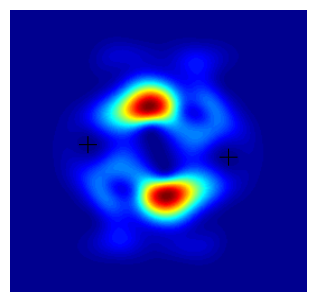

$t=10$

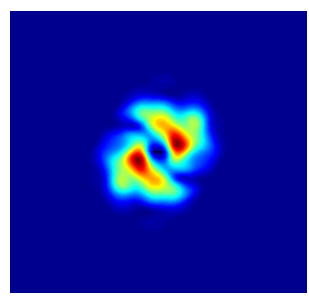

$t=0$

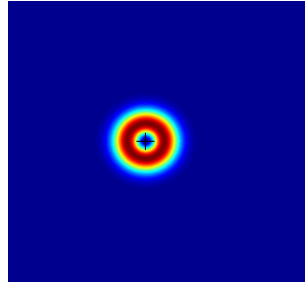

$t=1$

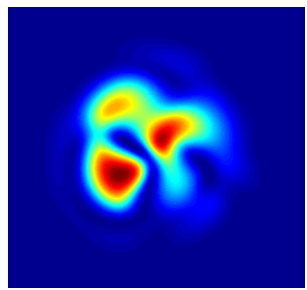

$t=5$

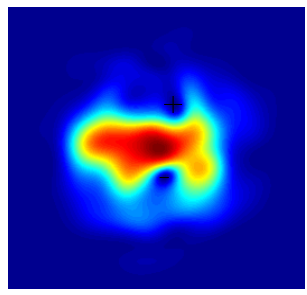

$t=10$

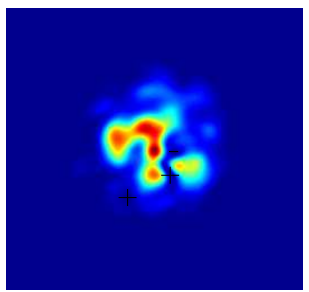

$t=0$

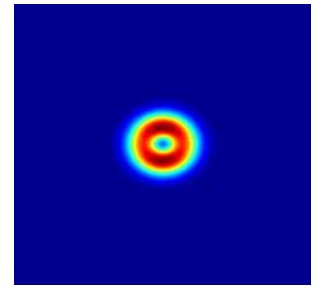

$t=1$

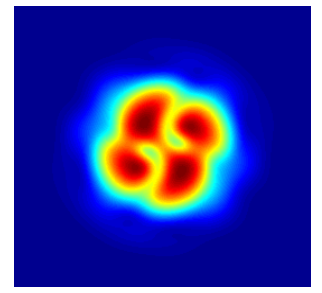

$t=5$

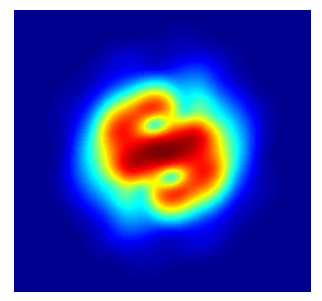

$t=10$

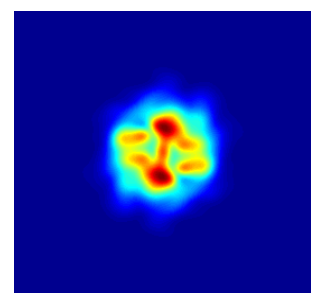

FIG. 7. Contour plots of the density $\rho_{-1}(t), \rho_{0}(t), \rho_{1}(t)$ and $\rho(t)$ (from left column to right column) over the dimensionless domain $[-8,8] \times[-8,8]$ in Example 4 with $\Omega=0.6, \beta_{n}=100$ and $\beta_{s}=90$ for case II.

CGPEs when the initial data is chosen as a stationary state with its center-of-mass shifted away from the external trap center. Along the numerical front, we discussed numerical methods for solving the CGPEs and applied them to study numerically the dynamics of spin-1 BEC. Our numerical results confirm the analytical results of CGPEs for the dynamics of spin-1 BEC.

Acknowledgments. The authors acknowledge support from Ministry of Education of Singapore grants R-146-000-120-112 and R-158-000-002-112. Y. Z. acknowledges support from the US Department of Energy under grant DE-FG02-05ER25698. We also thank the referees for their valuable comments and suggestion for improving the paper. 


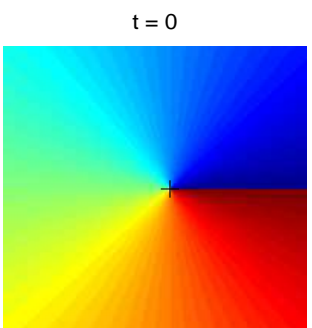

$\mathrm{t}=1$

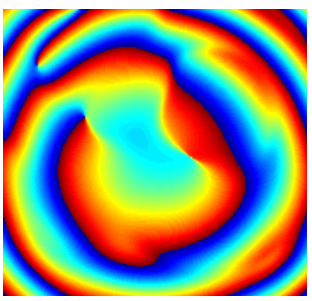

$t=5$

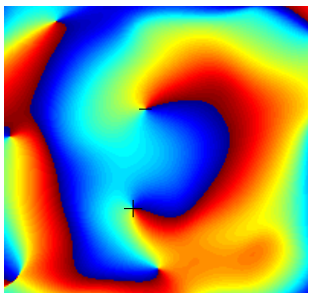

$t=10$

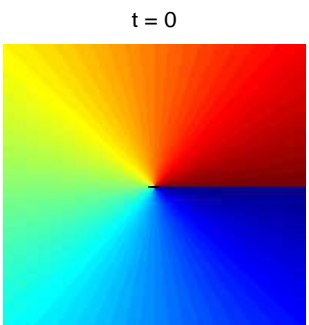

$t=1$

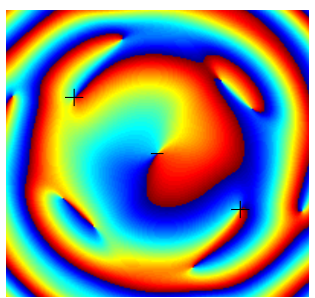

$t=5$

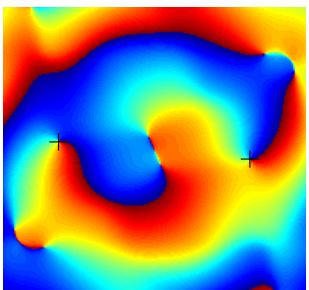

$t=10$

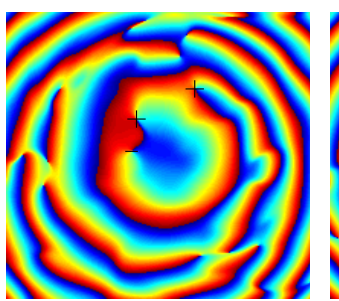

$t=0$

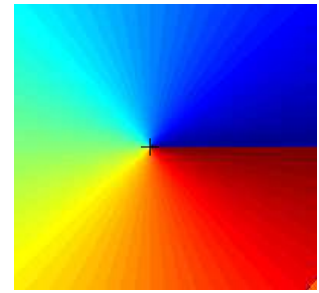

$t=1$

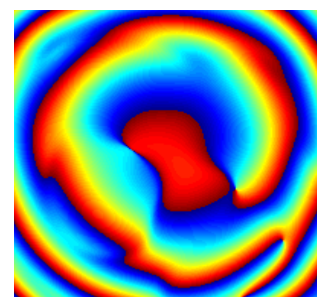

$\mathrm{t}=5$

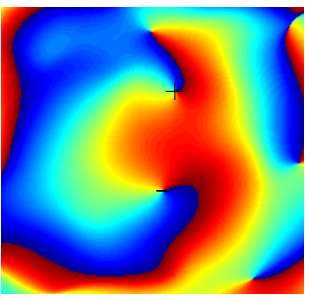

$t=10$

FIG. 8. Contour plots of the phase $S_{-1}(t), S_{0}(t)$ and $S_{1}(t)$ (from left column to right column) over the dimensionless domain $[-6,6] \times[-6,6]$ in Example 4 with $\Omega=0.6, \beta_{n}=100$ and $\beta_{s}=90$ for case II.

\section{REFERENCES}

[1] M. H. Anderson, J. R. Ensher, M. R. Matthews, C. E. Wieman and E. A. Cornell, Observation of Bose-Einstein condensation in a dilute atomic vapor, Science, 269 (1995), pp. 198-201.

[2] W. BAO, Analysis and efficient computation for the dynamics of two-component Bose-Einstein condensates, Contemporary Mathematics, 473 (2008), pp. 1-26.

[3] W. BaO, Q. Du AND Y. Zhang, Dynamics of rotating Bose-Einstein condensates and their efficient and accurate numerical computation, SIAM J. Appl. Math., 66 (2006), pp. 758786.

[4] W. BaO, H. Li and J. Shen, A generalized-Laguerre-Fourier-Hermite pseudospectral method for computing the dynamics of rotating Bose-Einstein condensates, SIAM J. Sci. Comput., 31 (2009), pp. 3685-3711.

[5] W. BAO AND F. Y. LiM, Computing ground states of spin-1 Bose-Einstein condensates by the normalized gradient flow, SIAM J. Sci. Comput., 30 (2008), pp. 1925-1948. 

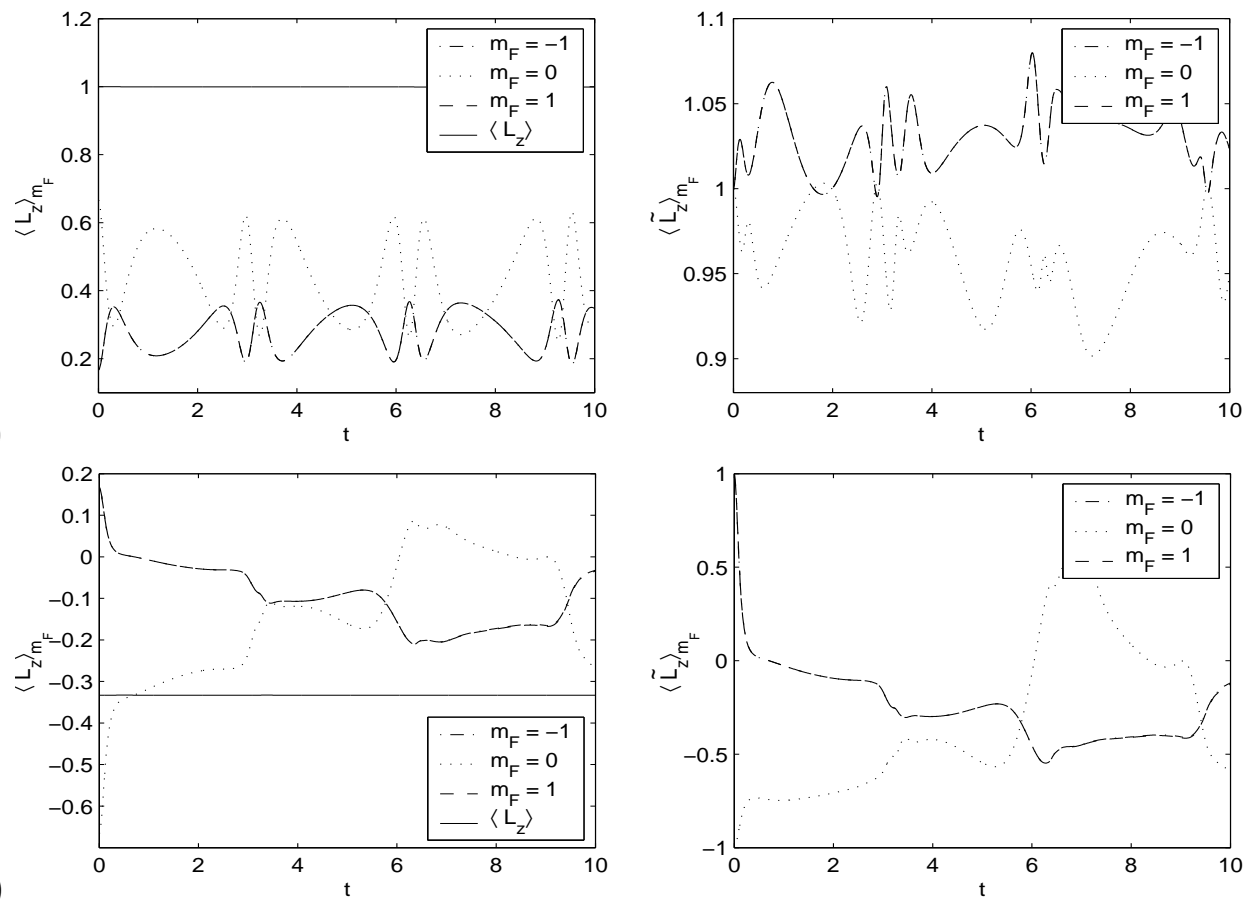

FIG. 9. Time evolution of the angular momentum expectation in Example 4 with $\Omega=0.6$, $\beta_{n}=100$ and $\beta_{s}=90$ : a) for case I; and b) for case II.

[6] W. BaO AND F. Y. Lim, Numerical methods for computing the ground state of spin-1 BoseEinstein condensates in uniform magnetic field, Phys. Rev. E, 78 (2008), article 066704.

[7] W. BaO, D. JaKsch, And P. A. Markowich, Numerical solution of the Gross-Pitaevskii equation for Bose-Einstein condensation, J. Comput. Phys., 187 (2003), pp. 318-342.

[8] W. Bao, P. A. Markowich, C. Schmeiser and R. M. Weishaupl, On the Gross-Pitaevskii equation with strongly anisotropic confinement: formal asymptotics and numerical experiments, Math. Models Meth. Appl. Sci., 15 (2005), pp. 767-782.

[9] W. BAO AND H. WANG, A mass and magnetization conservative and energy diminishing numerical method for computing ground state of spin-1 Bose-Einstein condensates, SIAM J. Numer. Anal., 45 (2007), pp. 2177-2200.

[10] W. BaO And Y. Zhang, Dynamics of the ground state and central vortex states in BoseEinstein condensation, Math. Models Meth. Appl. Sci., 15 (2005), pp. 1863-1896.

[11] I. Bialynicki-Birula and Z. Bialynicki-Birula, Center-of-mass motion in the many-body theory of Bose-Einstein condensates, Phys. Rev. A, 65 (2002), article 063606.

[12] C. C. Bradley, C. A. Sackett, J. J. Tollett and R. G. Hulet, Evidence of Bose-Einstein condensation in an atomic gas with attractive interaction, Phys. Rev. Lett., 75 (1995), pp. $1687-1690$.

[13] E. N. Bulgakov and A. F. Sadreev, Vortex phase diagram of $F=1$ spinor Bose-Einstein condensates, Phys. Rev. Lett., 90 (2003), article 200401.

[14] M. Caliari And M. SQuassina, Numerical computation of soliton dynamics for NLS equations in a driving potential, Electron. J. Diff. Equ., 2010:89 (2010), pp. 1-12.

[15] M. Chang, Q. Qin, W. Zhang, L. You And M. S. Chapman, Coherent spinor dynamics in a spin-1 Bose condensate, Nature Phys., 1 (2005), pp. 111-116.

[16] K. B. Davis, M. -O. Mewes, M. R. Andrews, N. J. Vam Druten, D. S. Durfee, D. M. Kurn And W. Ketterle, Bose-Einstein condensation in a gas of sodium atoms, Phys. Rev. Lett., 75 (1995), pp. 3969-3973.

[17] J. J. Garcia-Ripoll and V. M. Perez-Garcia and V. Vekslerchik, Construction of exact solutions by spatial translations in inhomogeneous nonlinear Schrödinger equations, Phys. Rev. E, 64 (2001), article 056602.

[18] E. P. Gross, Structure of a quantized vortex in boson systems, Nuovo. Cimento., 20 (1961), 
pp. $454-477$.

[19] T. L. Ho And V. B. Shenoy, Binary mixtures of Bose condensates of alkali atoms, Phys. Rev. Lett., 77 (1996), pp. 3276-3279.

[20] T. Isoshima, K. Machida And T. Ohmi, Spin-domain formation in spinor Bose-Einstein condensation, Phys. Rev. A, 60 (1999), pp. 4857-4863.

[21] A. Klein, D. JaKsch, Y. Zhang and W. BAO, Dynamics of vortices in weakly interacting Bose-Einstein condensates, Phys. Rev. A, 76 (2007), article 043602.

[22] J. Kronjäger, C. Becker, M. Brinkmann, R. Walser, P. Navez, K. Bonges and K. SENGSTOCK, Evolution of a spinor condensate: coherent dynamics, dephasing and revivals, Phys. Rev. A, 72 (2005), article 063619.

[23] H. J. Miesner, D. M. Stamper-Kurn, J. Stenger, S. Inouye, A. P. Chikkatur and W. Ketterle, Observation of metatable states in spinor Bose-Einstein condensates, Phys. Rev. Lett., 82 (1999), pp. 2228-2231.

[24] T. Mizushima, K. Machida And T. Kita, Axisymmetric versus nonaxisymmetric vortices in spinor Bose Einstein condensates, Phys. Rev. A, 66 (2002), pp. 053610.

[25] J. Mur-Petit, Spin dynamics and structure formation in a spin-1 condensate in a magnetic field, Phys. Rev. A, 79 (2009), article 053603.

[26] J. Mur-Petit, M. Guilleumas, A. Polls, A. Sanpera, M. Lewenstein, K. Bongs and K. Sengstock, Dynamics of $F=1{ }^{87} R b$ condensates at finite temperatures, Phys. Rev. A, 73 (2006), article 013629.

[27] O. E. Müstecaphoglu, M. Zhang and L. You, Tunneling of condensate magnetization in a double-well potential, Phys. Rev. A, 71 (2005), article 053616.

[28] T. Ohmi And K. MachidA, Bose-Einstein condensation with internal degrees of freedom in alkali atom gases, J. Phys. Soc. Jpn., 67 (1998), pp. 1822-1825.

[29] L. P. PitaevskiI, Vortex lines in an imperfect Bose gas, Soviet Phys. JETP, 13 (1961), pp. 451454.

[30] L. P. Pitaevskit and S. Stringari, Bose-Einstein condensation, Clarendon Press, 2003.

[31] J. Stenger, S. Inouye, D. M. Stamper-Kurn, H. J. Miesner, A. P. Chikkatur and W. Ketterle, Spin domians in ground-states Bose-Einstein condensates, Nature (London), 396 (1998), pp. 345-348.

[32] G. Strang, On the construction and comparison of difference schemes, SIAM J. Numer. Anal., 5 (1968), pp. 505-517.

[33] M. Uchiyama, J. Ieda and M. Wadati, Dark solitons in $F=1$ spinor Bose-Einstein condensate, J. Phys. Soc. Jpn., 75 (2006), article 064002.

[34] H. WANG, A time-splitting spectral method for computing dynamics of spinor $F=1$ BoseEinstein condensates, Int. J. Comput. Math., 84 (2007), pp. 925-944.

[35] Y. Zhang, Numerical study of vortex interactions in Bose-Einstein condensation, Commun. Comput. Phys., 8 (2010), pp. 327-350.

[36] Y. Zhang And W. Bao, Dynamics of the center of mass in rotating Bose-Eintein condensates, Appl. Numer. Math., 57 (1007), pp. 697-709.

[37] Y. Zhang, W. BAO AND H. Li, Dynamics of rotating two-component Bose-Einstein condensates and its efficient computation, Physica D, 234 (2007), pp. 49-69.

[38] W. Zhang, S. Yi AND L. You, Mean field ground states of a spin-1 condensate in a magnetic field, New J. Phys., 5 (2003), pp. 77-89.

[39] W. Zhang and L. You, An effective quasi-one-dimensional description of a spin-1 atomic condensate, Phys. Rev. A, 71 (2005), article 025603. 